\title{
Hypersonic Wind Tunnel Calibration Using the Modern Design of Experiments
}

\author{
Matthew N. Rhode ${ }^{*}$ and Richard DeLoach. ${ }^{\dagger}$ \\ NASA Langley Research Center, Hampton, Virginia 23681
}

\begin{abstract}
A calibration of a hypersonic wind tunnel has been conducted using formal experiment design techniques and response surface modeling. Data from a compact, highly efficient experiment was used to create a regression model of the pitot pressure as a function of the facility operating conditions as well as the longitudinal location within the test section. The new calibration utilized far fewer design points than prior experiments, but covered a wider range of the facility's operating envelope while revealing interactions between factors not captured in previous calibrations. A series of points chosen randomly within the design space was used to verify the accuracy of the response model. The development of the experiment design is discussed along with tactics used in the execution of the experiment to defend against systematic variation in the results. Trends in the data are illustrated, and comparisons are made to earlier findings.
\end{abstract}

\section{Nomenclature}

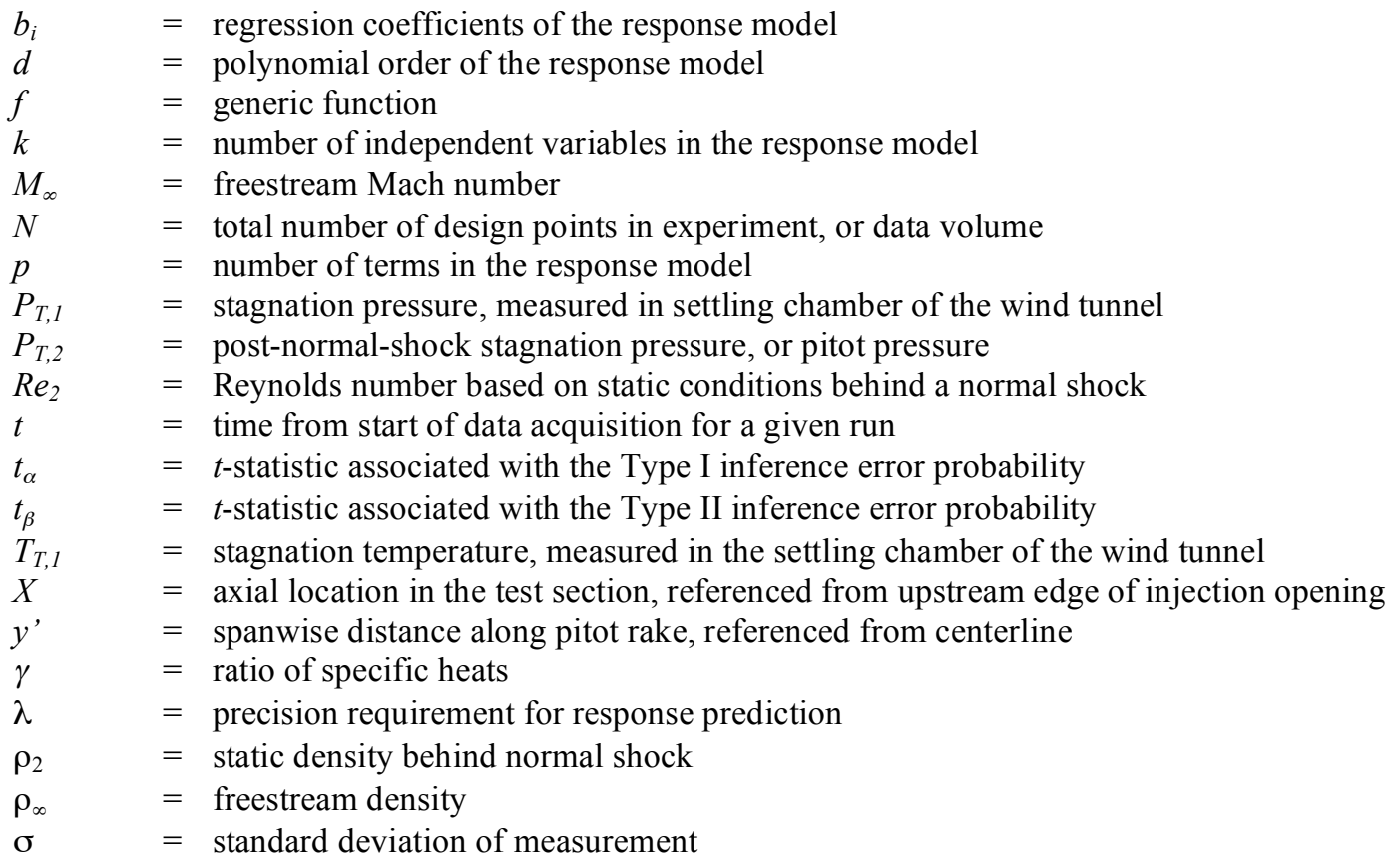

\section{Introduction}

$\mathrm{N}$ recent years, there has been increasing emphasis placed on the determination and documentation of uncertain1 ties for measurements made in ground-based test facilities, with many papers reported in the literature as well as the publication of guides and standards. ${ }^{1,2}$ This emphasis is due not only to a better understanding of the topic by a wider segment of the aerospace community, but also to the need for more accurate measurements as design and cost margins become slimmer. Many aerospace projects from yesteryear enjoyed comprehensive wind tunnel test pro-

\footnotetext{
* Aerospace Engineer, Aerothermodynamics Branch, Mail Stop 408A, AIAA Senior Member.

${ }^{\dagger}$ Senior Research Scientist, Aeronautics Systems Engineering Branch, Mail Stop 238, AIAA Senior Member.
} 
grams that often involved experiments with multiple test articles in several different facilities. The large volumes of data coupled with various comparisons amongst models and facilities often allowed engineers to sort out questionable data - outlier data from a particular model, say, or problems with measurements in a given facility - and base results on multiple experiments performed over a longer period of time. Projects today, however, are more likely to be fast-paced, with fewer personnel and smaller resource budgets. Test programs may consist of just one or two experiments with a single wind tunnel model and little-to-no overlap between facilities. With no separate verification of the results, it is important that the quality of the data from those few tests is well understood. To be fair, computational fluid dynamic (CFD) solutions are now often employed to independently check the results from ground-based tests. But the number of CFD cases is frequently restricted to just a few conditions by the same limited budgets.

Fortunately, there have been marked improvements in data quality assurance for wind tunnel measurements. Uncertainty analysis infrastructures have been emplaced at many facilities for use in pre-test and post-test estimations of data precision. ${ }^{3,4}$ Software programs even allow the computation of uncertainty values during data reduction following a wind tunnel run. ${ }^{5}$ Most of these analyses are based on error propagation methods, ${ }^{6}$ where the uncertainty of a quantity is calculated from the uncertainties of individual contributing sources, such as various instrumentation, the model attitude, test techniques, and the test article. One contributing source not often appreciated and often overlooked is the variance in the flow conditions. As shown by Kammeyer, ${ }^{7}$ the uncertainty in a calculated pressure or drag coefficient can be dominated by the uncertainty in the Mach number, with much less contribution coming from the force balance, pressure transducer, or other sources. The uncertainty in the flow conditions stems from two sources: the calibration of the facility itself and spatial variations in the flow. With the former, the uncertainty is often derived from the standard error of whatever regression model is fit to the data, and it can be reduced by replicating measurements and improving the functionality of the model. The spatial variations in the flow are the result of acoustic noise and disturbances emanating from irregularities in the nozzle contour, due either to manufacturing errors or an imperfect nozzle design. These variations are generally repeatable and cannot be removed or minimized through repeated measurements. The effects of spatial non-uniformity on measurements made during a wind tunnel experiment can be hard to define and depend on the magnitudes and locations of disturbances relative to a test article of a particular shape and orientation to the flow. In uncertainty analyses of the type described above, the uncertainty in flow conditions from the two sources tend to be treated as separate quantities. Beyond designing and/or fabricating a new wind tunnel nozzle, there is not much that can be done to lessen the spatial non-uniformity in the flow. But we can reduce the calibration uncertainty through improved experiments and by acquiring more data, either through larger and more involved experiments, or by performing smaller but more frequent calibrations over a span of time.

Wind tunnel calibrations, however, tend to occur infrequently due in large part to the time and resources required to perform a calibration, coupled with the fact that calibrations must compete with production/research testing for finite resources and are often deferred for that reason. Intervals of several years, commonly, may pass between events, and potential changes in the flow characteristics of the facility may go unnoticed during that time. For this reason, the "smaller but more frequent" option is preferred, since this also provides a check on the long-term stability of the flow properties as well as an indication of any sudden changes. Such checks dovetail nicely with the use of statistical process control (SPC) methods ${ }^{8,9}$ to track results over time and establish long-term means and uncertainties on values. Because resources are limited, though, frequent facility calibrations necessitate the use of relatively compact experiments, as opposed to the usual calibrations that often involve hundreds of runs and take weeks or months to accomplish.

The Modern Design of Experiments (MDOE) provides a means to develop highly efficient experiment designs that can be tailored to the specific problem, yielding results to within a specified precision. MDOE is a unified experiment design, execution, and analysis process introduced at the Langley Research Center (LaRC) for use in wind tunnel tests and also for experiments in a wide array of laboratory settings, with a typical resource savings of a factor of two or more. ${ }^{10,11}$ An extension of industrial experiment design methods to scientific applications, MDOE has seen increasing use throughout the aerospace community for numerous ground-based experiments, and was applied recently to the calibration of the NASA LaRC 20-Inch Mach 6 Tunnel, a workhorse hypersonic facility in the Langley Aerothermodynamic Laboratory (LAL). The 20-Inch Mach 6 Tunnel had not been calibrated in several years, so a new calibration was desired not only as an update, but also to expand the calibration over a wider range of the facility's operating envelope. This experiment served as a trial case for a strategy of improving the precision in results from LAL wind tunnels through more frequent and accurate facility calibrations and the subsequent reduction of flow condition uncertainties. 


\section{Description of Facility}

The 20-Inch Mach 6 Tunnel is a blowdown wind tunnel that uses dry air as the test gas. ${ }^{12,13}$ Air is transferred from a high-pressure bottlefield to a 600-psia reservoir where the air is heated to a maximum temperature of $1000 \mathrm{R}$ by an electrical resistance heater. Two inline, sintered-metal filters are located between the heater and the settling chamber to reduce the number particles in the flow. The upstream filter is capable to capturing particles larger than $20 \mu \mathrm{m}$ in size, and the second filter is rated at $5 \mu \mathrm{m}$. The settling chamber, rated at a maximum operating pressure of 525 psia, contains a perforated conical baffle at the entrance and internal screens to aid flow mixing and reduce turbulence in the air stream. From the settling chamber, the air expands through a non-cooled, two-dimensional contoured nozzle and into a test section 20 by 20.5 inches in cross section. The nozzle has a throat 0.339 inches in height by 20 inches in width, contoured upper and lower walls, and flat sidewalls. The upstream end of the test section is located 81.4 inches from the throat, and the usable test section length is 48.4 inches. An adjustable second minimum is located downstream of the test section to improve pressure recovery. The flow exhausts through an aftercooler and into a combination of 41,60 , and 100 -ft-diameter vacuum spheres, or to the atmosphere when using an annular air ejector. Maximum run times range from 2 to 15 minutes depending on the operating conditions and the available vacuum capacity. ${ }^{\ddagger}$ A photograph of the facility is shown in Fig. 1 and a schematic of the settling chamber and test section are given in Fig. 2.

Models are mounted to the model support system (MSS) using a variety of sting and strut hardware. The MSS consists of an arc sector connected to a hydraulic injection system located in a housing below the closed test section. The computer-controlled system can provide pitch angles from -5 to $+55^{\circ}$ and yaw angles up to $\pm 8^{\circ}$. The injection system is used to place a test article into the flow following flow establishment and to remove the test article prior to flow stoppage, in both instances preventing strong dynamic starting loads on the model. Injection speed can be varied to provide injection times ${ }^{\S}$ from 0.5 to 1.2 seconds, depending on the test requirements. A floor plate attached to the MSS fills the opening in the lower test section wall when a model is injected into the tunnel. A slot is provided in the floor plate for a strut and its

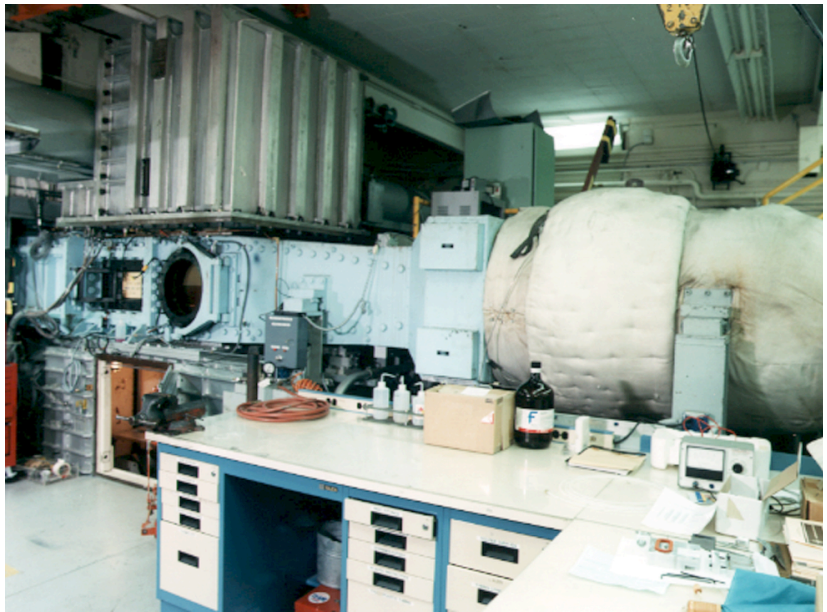

Figure 1. External view of 20-Inch Mach 6 Tunnel. associated pitch and yaw movements. A number of filler plates are available to close off portions of this slot depending on the required range of strut movement. The floor plate can be removed from the MSS and replaced with a custom part if needed for a specific test.

The facility operates at stagnation pressures from 15 to 525 psia and stagnation temperatures up to $960 \mathrm{R}$. In practical usage, however, a supply line mass flow limit of $60 \mathrm{lbm} / \mathrm{sec}$ restricts the maximum stagnation pressure to approximately 490 psia. Similarly, the stagnation temperature is operationally limited to $935 \mathrm{R}$ to prolong the life of the electrical resistance heater and to reduce maintenance on valves and other hardware. Over these pressure and temperature ranges, the freestream Mach number varies from 5.75 to 6.05 , with a unit Reynolds number from 0.3 to $8.5 \times 10^{6}$ per foot. An activated alumina dryer provides dew point temperature of $419 \mathrm{R}$ at a pressure of $600 \mathrm{psia}$.

The test section is equipped with five windows to provide optical access for a variety of flow visualization and optical thermography techniques. Two sets of windows are located in the sidewalls of the test section and are generally used for schlieren visualization. The forward windows are 17 inches in diameter and located near the center of rotation of the arc sector. The aft windows are $17 \times 9$ inches in size and are positioned 23.44 inches aft of the forward window centerline. Parabolic mirrors, suspended from a traversable overhead beam, are positioned on opposite sides of each set of windows to provide simultaneous schlieren visualization. A 17.5 x 11.5-inch window is located on top of the test section and is frequently used for phosphor thermography. The window can be replaced

\footnotetext{
* Other facilities utilize the same air storage and vacuum spheres, so the full capacity of either may not be available at a given time.

${ }^{\S}$ Injection time is the time required for the full injection stroke of 39.5 inches.
} 
with a metal blank, a metallic frame holding 5 or 8-inch-diameter infrared windows, or one of two enclosures containing injectable probes or a three-axis flowfield survey mechanism.

The 20-Inch Mach 6 Tunnel has a stand-alone data system with hardware and software identical to other facilities in the Langley Aerothermodynamics Laboratory (LAL). The core of the system is a 256-channel, amplifier-perchannel data acquisition system manufactured by the NEFF Instrument Corporation. Each channel contains programmable gains and filters, with signals multiplexed to a 16-bit analog-to-digital converter. A signal conditioner provides up to \pm 10 volts excitation to various instruments. A typical sampling rate is 50 samples per second for each channel, although much higher rates are possible. Presently, 512 channels of pressure measurements are available via electronically scanned pressure (ESP) sensors using a Pressure Systems, Inc. System 8400. ESP modules containing up to 64 sensors and of various pressure ranges are on hand for use in the facility, and other pressure instrumentation is available for use in certain applications. Two PC-type computers are used to control the data system; one is dedicated to data acquisition while the other is used for data reduction. Data are typically available only minutes following a run.

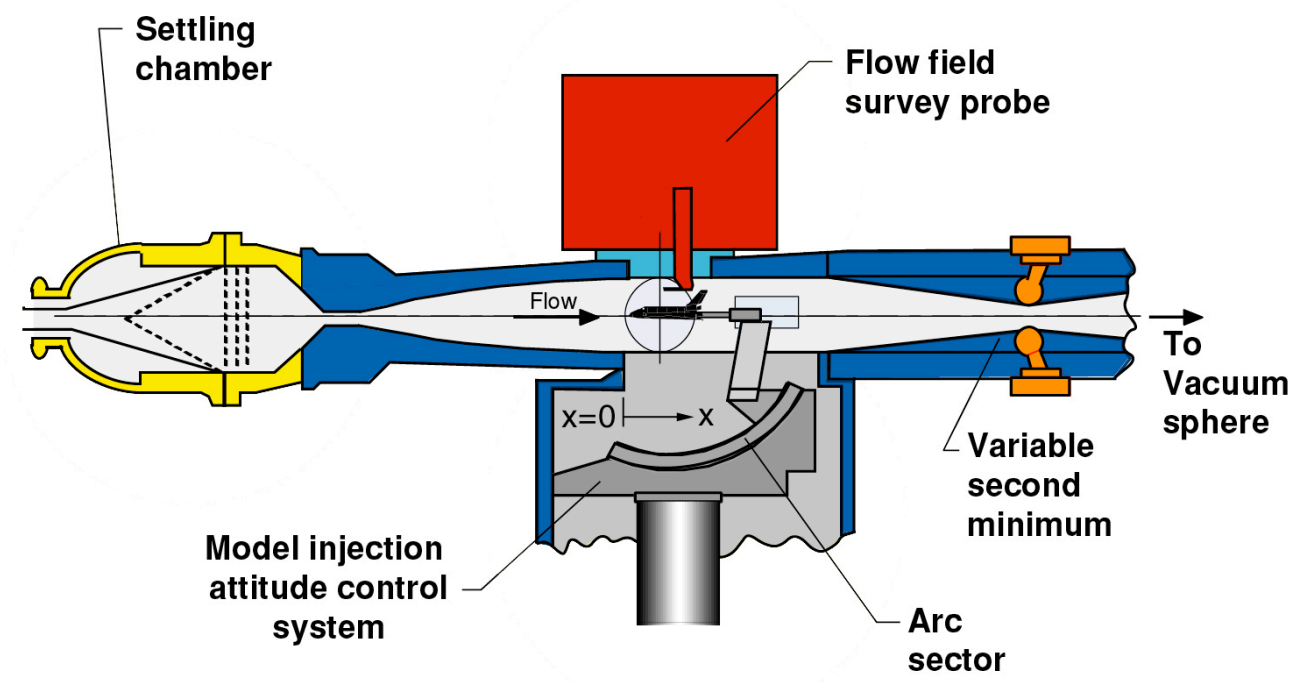

\begin{abstract}
Nominal Mach number: Reynolds number (x 106/ft): Dynamic pressure (psf):
\end{abstract}

\section{0}

0.35 to 8.5

55 to 1110

\begin{abstract}
Total pressure (psia): $\quad 20$ to 480 Total temperature $\left({ }^{\circ} \mathrm{R}\right): \quad 805$ to 960 Run time (minutes):
\end{abstract}

Figure 2. Schematic of settling chamber and test section.

\section{Calibration Methodology}

In planning for a facility calibration, one must first decide what information is of interest and how that information will be obtained, and to what accuracy it needs to be known. This establishes the scope of the experiment as well as instrumentation and hardware requirements. Typical objectives in a wind tunnel calibration may be to determine Mach number or pitot pressure as a function of stagnation conditions, location within the test section, nozzle shape (for variable Mach number facilities), and other parameters. ${ }^{14}$ It is commonplace to investigate the flow spatial uniformity, with particular attention given to the presence of embedded shocks or other disturbances. Other factors of interest may include core flow size, run time, power usage and/or operating costs, total temperature recovery and spatial uniformity, flow angularity, freestream turbulence levels, effects of environmental conditions, blockage, operating limits, etc. The calibration of a new facility or one that has seen extensive refurbishment may wish to examine many or all of these factors, as there would be little or no previous data to draw on. A longstanding facility might require a more limited calibration as an update to previous information, and thus the calibration could be narrower in scope.

The recent calibration of the 20-Inch Mach 6 Tunnel was undertaken to expand the calibration over a wider operating range of the facility and to update the previous calibration that was several years old. Because the nozzle 
had not been modified, there was no expected change in the amount of core flow variation or in the presence and location of known disturbances. Thus the experiment need not focus on locating and tracing flow disturbances, but rather on revising the functional response of Mach number or pitot pressure to a broader range of operating conditions. ${ }^{* *}$ Likewise, total temperature recovery had been investigated in the past using a rake of shielded total temperature probes, with results showing little spatial variation in total temperature as measured in the test section, indicating good thermal mixing in the settling chamber of the tunnel. Although temperature recovery and spatial variation are important pieces of information, they were not deemed as necessary to measure at this time. Therefore, the current calibration of the 20-Inch Mach 6 Tunnel was limited to updating the response of the flow conditions as a function of the stagnation conditions and the axial location in the test section (to assess axial Mach number gradients).

In most supersonic wind tunnel facilities, flow conditions are calculated based on measured values of stagnation pressure and temperature in the settling chamber and a calibrated value of freestream Mach number in the test section. Mach number itself is computed from measured quantities and can be determined in a number of ways. Beyond a value of 1.6, Mach number is most accurately computed ${ }^{15}$ from the ratio of pitot pressure to total pressure, given by the following gas dynamic equation. ${ }^{16}$

$$
P_{T_{2}} / P_{T_{1}}=\left[\frac{(\gamma+1) M_{\infty}^{2}}{(\gamma-1) M_{\infty}^{2}+2}\right]^{\frac{\gamma}{\gamma-1}}\left[\frac{\gamma+1}{2 \gamma M_{\infty}^{2}-(\gamma-1)}\right]^{\frac{1}{\gamma-1}}
$$

Mach number can also be computed from the ratios of wall static to total pressure and wall to pitot pressure (the Rayleigh pitot tube formula), but these methods are less reliable at higher Mach numbers due to the difficulty in accurately measuring low wall static pressure values, especially in hypersonic wind tunnels. Moreover, at hypersonic Mach numbers, the measured wall pressure might not represent the freestream static value since transverse pressure gradients may exist in thick hypersonic boundary layers. Other means of deriving the freestream Mach number include measured shock wave angles from schlieren photographs and surface pressure measurements on cones or wedges in the test section flow. These alternate methods are seldom utilized as the primary means to calibrate a facility, but can be useful as checks on the main results. ${ }^{15}$

In the facilities of the LAL, it is standard practice to calibrate the pitot pressure, $P_{T, 2}$, as a function of the variables of interest rather than Mach number since $P_{T, 2}$ is a measured quantity and its uncertainty can be directly applied to various uncertainty analyses for operational tests. Mach number is never measured directly; it is merely calculated from other measured values. As will be shown in the next section, for the 20-Inch Mach 6 Tunnel and other facilities in the LAL, $P_{T, 2}$ is a low-order function of the stagnation conditions in the settling chamber and thus can be calibrated accurately in relatively few runs.

\section{Design of the Calibration Experiment}

The objective of the 20-Inch Mach 6 calibration was to acquire sufficient data to create a response surface model that could be used to predict the average pitot pressure across the core flow of the test section, to within a specified uncertainty, as a function of the measured stagnation pressure and temperature in the settling chamber and the axial location within the test section. To determine what is "sufficient data", two basic questions must be answered: how many data points are required, and where in the design space should the points be located? Both questions are addressed in this section as we discuss the test matrix design in detail.

\section{A. Establishing the Design Space}

Before either of the questions posed above can be answered, we must first identify the ranges of independent variables, or the design space, over which the calibration will occur, as the choice of the design space can affect both the number of points and their locations. For example, over a narrow range for a given factor, the response variable may exhibit a low-order trend, whereas over a wider range of that factor, the response may be highly non-linear, requiring many more points in order to fit an adequate response surface. The shape of the design space has an effect

\footnotetext{
${ }^{* *}$ While the focus may be on updating the response function, the method of calibration - use of a pitot rake - allows the concomitant assessment of flow spatial variation at the design points in the experiment.
} 
as well. Many well-established experiment designs with desirable properties exist for spherical and cuboidal design spaces and are frequently used. Irregularly shaped design spaces or ones with constraints may require the use of a custom, computer-generated design for optimal point distribution. ${ }^{17}$ Thus, we must start with determining the range of factors for the calibration design.

For the 20-Inch Mach 6 Tunnel, we desired to calibrate over as wide a range of the region of operability as would be useful for future fluid dynamic experiments in the facility. By operating at lower stagnation temperatures, operating conditions can be attained more quickly during a run, saving not only on run time, but also the pumpdown time between runs, as less total air flows into the vacuum spheres during a run. The lower temperatures should also reduce thermal effects on the test article and instrumentation, particularly beneficial for force and moment testing. The region of operability for the facility is defined by a number of mechanical, thermal, and fluid dynamic constraints, as noted in Figure 3. The electrical resistance heater limit fixes the upper boundary for the stagnation temperature, while the supply line mass flow rate limit effectively defines the upper stagnation pressure limit as a function of temperature. The minimum stagnation pressure is approximately $15 \mathrm{psia}$, as it is difficult to start and maintain flow in the facility at lower pressures.

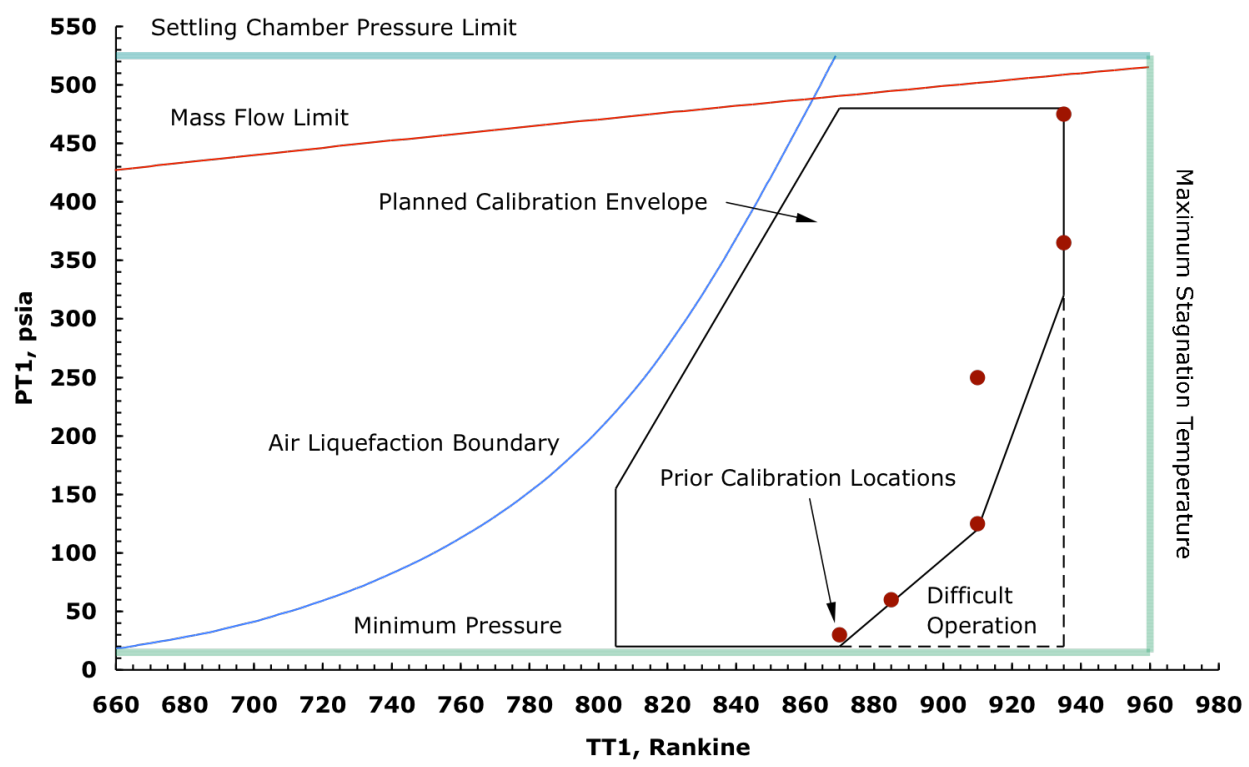

Figure 3. Operating envelope for the 20-Inch Mach 6 Tunnel

Within this region of operability is our region of interest that we choose as the design space. The design space pressure limits are close to the operability limits, but have been backed off a bit to ensure reliable operation over the calibrated range. The maximum stagnation temperature was set at $935 \mathrm{R}$ in order to prolong the life of the heater bundle and various tunnel hardware. Note the constraint boundaries in the area of low pressures and elevated temperatures. The low mass flow rates at these conditions, coupled with the thermal mass of the inline filters, make it difficult to obtain the temperature in the settling chamber before the vacuum sphere pressure builds up to levels where flow can no longer be sustained or the remaining useful run time would be insufficient for most tests. The linear nature of these boundaries is an artifact of the particular software used to design this experiment. The theoretical air liquefaction line largely governs the lower temperature boundary of the design space, and a margin of approximately $10 \mathrm{R}$ from the liquefaction limit was maintained. The minimum stagnation temperature for the calibration was set at $805 \mathrm{R}$. The tunnel can be operated at lower temperatures; however, this would have increased the amount of thermal cycling on the facility since the test matrix would be run in a randomized order. Deemed a safety issue, the thermal cycling was reduced to an acceptable level by raising the minimum stagnation temperature.

The nominal stagnation pressure and temperature settings for the previous 1995 calibration are also plotted in Fig. 3. The temperature was maintained, more or less, on the upper operational boundary in order to provide a high 
thermal driver ${ }^{\dagger \dagger}$ for aeroheating tests in the facility. Note that since the stagnation temperature was varied with pressure, the effects of each on the response were confounded with one another. That is, the independent effect of temperature cannot be separated from the pressure effect, and vice versa. The present calibration spans a much greater extent of the pressure-temperature envelope, and the design point placement - to be shown later - allows independent assessment of the pressure and temperature effects and any interaction between the two.

The third independent factor in the experiment is the axial location within the test section, denoted as $X$. The axial location is referenced from the upstream edge of the opening for the injection system in the lower test section wall ( $X=0$, see Fig. 2). Depending on the vertical location of a model in relation to the arc sector center of rotation, a model can translate slightly forward when pitched to negative angles. For this reason, the forward limit for model axial location is generally kept around 1 inch from the upstream edge of the floor plate. Thus, the upstream design space boundary is $X=1 \mathrm{inch}$. The downstream boundary was set at $X=25$ inches, since the vast majority of models tested in the facility are sting mounted (either straight, dogleg, or blade) and therefore must stay some distance in front of the strut head. Note that in previous calibrations of the 20-Inch Mach 6 Tunnel, the axial location was referenced from the center of the forward set of schlieren windows. This was changed in the current calibration to provide a fixed reference location that was easier to measure from.

\section{B. Data Volume Requirements}

Now that we have defined the design space for the calibration, we need to determine the volume of data necessary to produce a response surface that adequately models the pitot pressure, $P_{T, 2}$, as a function of the independent variables. This is an important step in formally designed experiments, as too few data points will yield a response surface lacking the desired precision or functionality, and substantially more data points needlessly wastes resources. The necessary volume of data depends on five factors: (1) response model complexity, (2) intrinsic variance in the raw data, (3) required precision, (4) inference error risk tolerance, and (5) model confirmation "comfort level." Each of these will be discussed in the context of the current calibration.

The number of terms, $p$, in a $d^{\text {th }}$-order polynomial of $k$ independent variables is given as: ${ }^{18}$

$$
p=\frac{(d+k) !}{d ! k !}
$$

It can be seen by inspection of Eq. (2) that the number of terms in the polynomial grows rapidly as the order of the model and/or the number of variables increases. Thus, it is important to understand the expected functionality of the response so that an adequate response model can be described. Analysis of the previous calibration data gave insight into the behavior of the pitot pressure. Figure 4 shows a strong, near-linear dependence of $P_{T, 2}$ with stagnation pressure for a fixed axial location in the test section. Very little curvature is seen in the trend, indicating that a quadratic model would be appropriate. The prior calibration did not include the stagnation temperature as an independent variable so its effect cannot be determined from the data. However, the response due to changes in temperature is expected to be small, and due primarily to modification of the tunnel wall boundary layer thickness via changes in Reynolds number. The trends in $P_{T, 2}$ with axial location are shown in Fig. 5 for the previous calibration. Here, the change in pitot pressure from the forward-most axial location tested is plotted as a function of axial location for three stagnation

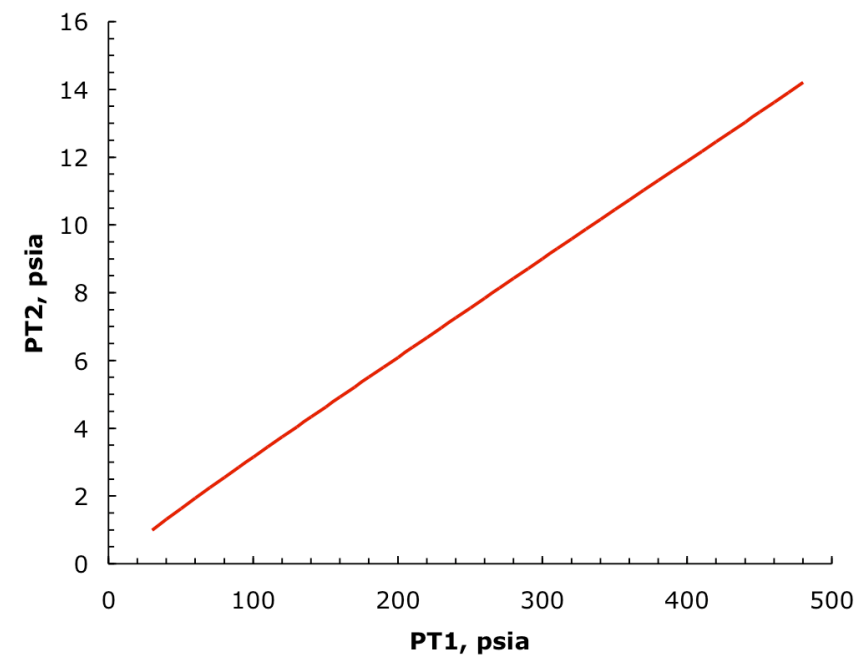

Figure 4. Effect of stagnation pressure on the pitot pressure, from the 1995 calibration.

\footnotetext{
${ }^{\dagger}$ The thermal driver is the difference between the stagnation temperature and the cold wall temperature on the wind tunnel test article. A high thermal driver reduces uncertainty in heat transfer measurements.
} 
pressure values spanning the calibrated range. (Again, note the change in origin for the axial location from the previous calibration to the current one.) The data show a pronounced quadratic trend with $X$, and the change in slope with stagnation pressure indicates the presence of an interaction between pressure and axial location. This result is unsurprising since most supersonic facilities have some axial Mach number gradient in the test section.

It is often a good idea, when resources permit, to increase the order of the response surface model over that of the expected response so that an assessment of any lack of fit in the model can be made. However, for $k=3$ independent variables, raising the order of the response surface model from quadratic $(d=2)$ to cubic $(d=3)$ doubles the number of terms in the model from 10 to 20 . Test section axial location was the only parameter where a pure quadratic trend was seen. But the lack of any inflection in the response of $P_{T, 2}$ with $X$ implies that a cubic term is not present. Therefore, a quadratic response surface model in the three independent variables was initially hypothesized and subsequently found to be adequate, as will be shown later.

The data volume can also be affected by the choice of the response variable. Many facilities use Mach number as the response, calibrating Mach number as a function of other parameters. Figure 6 shows Mach number as a function of $P_{T, 1}$ for the 1995 calibration. These are the same data as shown in Fig. 4, only couched in terms of Mach number, as calculated from the ratio, $P_{T, 2} / P_{T, 1}$. Analysis shows that a $4^{\text {th }}$-order polynomial would be required to reproduce this trend, as opposed to the near-linear response of $P_{T, 2}$ for the same data. From Eq. (2), the number of model terms required for a quartic response in three variables is 35 , compared to 10 terms needed for the quadratic model $-\mathrm{a}$ factor of 3.5. Hence, a tremendous savings in resources can be realized through careful selection of the response variable. A lower-order model can often be achieved by using, say, the logarithm or square root of a value rather than the value itself, for either the response or its

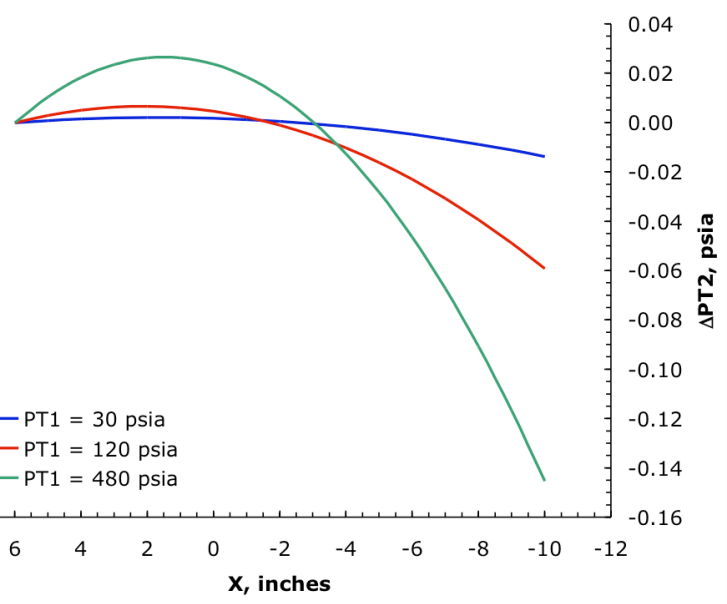

Figure 5. Trends in pitot pressure with test section axial location, from the 1995 calibration.

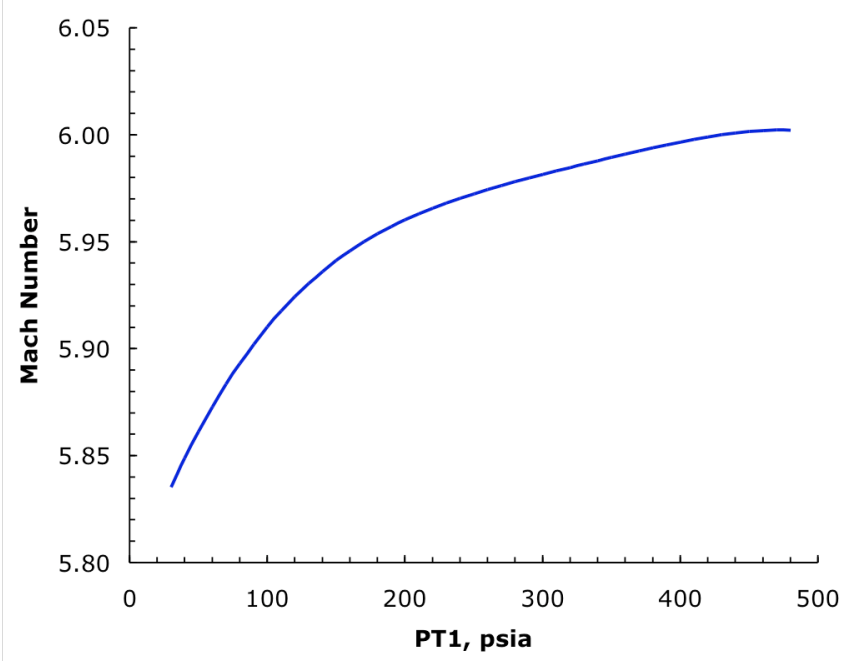

Figure 6. Mach number as a function of stagnation pressure, from the 1995 calibration.

independent variables. This is sometimes seen in wind tunnel calibrations, where the Mach number may be fit as a function of the logarithm of the stagnation pressure. ${ }^{19}$ Such a function was investigated for the present calibration, but the trend was found to remain at least cubic. It is well worth the time and effort to understand the physical and mathematical relationships between the response and the independent variables since a better choice of response model can result in considerable savings in the required data volume, as demonstrated here.

The quadratic response model for $P_{T, 2}$, the average pitot pressure across the core flow, is given by the following equation.

$$
P_{T_{2}}=b_{0}+b_{1} P_{T_{1}}+b_{2} T_{T_{1}}+b_{3} X+b_{4} P_{T_{1}} T_{T_{1}}+b_{5} P_{T_{1}} X+b_{6} T_{T_{1}} X+b_{7} P_{T_{1}}^{2}+b_{8} T_{T_{1}}^{2}+b_{9} X^{2}
$$

The independent variables are $P_{T, l}, T_{T, I}$, and $X$, and the $b_{i}$ 's are the model coefficients, determined via fitting the data by regression. The full quadratic model features an intercept, three first-order terms, three interaction terms, 
and three quadratic terms, for a total of ten terms altogether. Since one degree of freedom is required for every model coefficient, a minimum of 10 data points would be necessary for this experiment. However, acquiring only 10 points would force the response surface through each point and leave no additional degrees of freedom to assess the quality of the model. By obtaining more than the minimum 10 points, it is possible for the response surface to "float", which allows the quality of the fit to be estimated by examining the residuals. The question is, how many additional points are required?

The total number of points necessary to construct a $d^{\text {th }}$-order model in $k$ independent variables that will predict the response to within a specified tolerance is given as:

$$
N=\frac{(d+k) !}{d ! k !}\left(t_{\alpha}+t_{\beta}\right)^{2} \frac{\sigma^{2}}{\lambda^{2}}
$$

Here, $\sigma$ is the estimated standard deviation in the measurement, $\lambda$ is the precision requirement for the response surface, and $t_{\alpha}$ and $t_{\beta}$ are statistics related to acceptable inference error probabilities for Type I and Type II errors, respectively. From Eq. (4), we observe that the number of points, $N$, grows as the variance in the data increases; as the response model precision requirement increases; or as the $t$-statistics become larger with less tolerance for inference error. As will be seen, there is always a tradeoff between the desire for high precision and the limitations in resources.

From the 1995 calibration, the average variation in pitot pressure across the core flow was found to be approximately 0.025 psia. Requiring the prediction uncertainty to be at least equal to the average core flow variation $(\lambda=$ $\sigma$ ), and assuming $5 \%$ inference error probabilities (95\% confidence levels) for both error types, the total data volume given by Eq. (4) is $N=130$ points. This number of points would have called for test time well in excess of the limited resources available to perform this experiment, considering the expected decrease in run productivity from randomizing the run order. To bring the data volume down to manageable levels, we must allow either a larger tolerance for inference errors or a larger uncertainty in the predicted response, or both.

It would be appropriate here to discuss the two types of inference errors and how they affect the response surface model and its associated uncertainty. A Type I error occurs when we infer a difference between two results, say, when none exists, or when we include a response model term erroneously. A Type II error is failing to observe a true difference, or rejecting a response model term that truly exists. ${ }^{20}$ For reasonably low inference error probabilities, both types of error would involve terms that have small effects on the predicted response. That is, we are unlikely to reject a term having a strong effect on the response, or accept a term that has no effect whatsoever. Rather, we would be adding or omitting terms that cause little shift in the prediction from the model. However, Type I errors increase the uncertainty in the prediction since the degrees of freedom associated with the erroneously included terms are counted towards the model instead of the error. Therefore, adding unnecessary terms to a model adds to the uncertainty while producing little change in the predicted response. For Type II errors, the consequences are somewhat less. The failure to include one or two small terms will have little impact on the prediction, and the uncertainty will be affected to a lesser degree as well. Consequently, if we need to relax the inference error tolerances in order to reduce the data volume required to create a response model, we prefer to loosen the tolerance on the Type II error rather than the Type I. To note, some experiments require a greater ability to distinguish small effects, and thus the Type II error probability must be kept low to increase the resolving power. For the present calibration, the decision was made to leave the Type II error probability at $5 \%$ in order to capture all the model terms that may be statistically significant, relative to the error (i.e. able to be reliably distinguished from the noise). Any such terms having a negligible effect on the response could later be dropped in favor of simplifying the model with no real effect on the outcome.

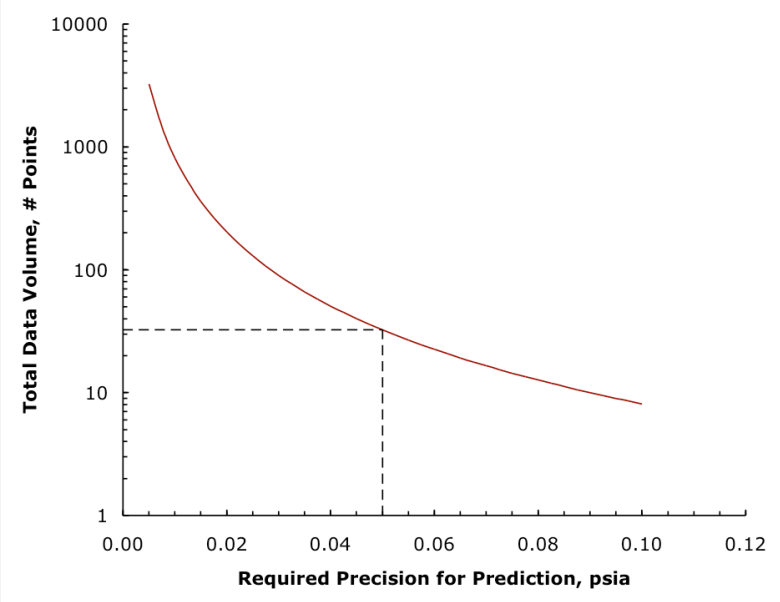

Figure 7. Data volume as a function of the required precision. 
The $t$-statistics associated with the inference error probabilities can be found from tables in any statistics text. Using these tabulated values, and the pitot pressure measurement uncertainty, $\sigma=0.025$ psia, we can see how the total data volume varies as a function of the prediction precision per Eq. (4). The trend is illustrated in Fig. 7. Note that the precision interval half-width is proportional to the square of the data volume, so to double the precision (halve the uncertainty interval) requires four times as much data. With the test time available for the experiment, and again noting the expected reduction in run productivity due to randomization of the test matrix, it was estimated that resources would be available for roughly 30-40 runs to construct the response surface, plus time needed to set up and "shake down" the experiment, as well as a series of confirmation points to validate the response surface. From Fig. 7, the estimated allowable data volume corresponds to a precision of approximately $\pm 0.05 \mathrm{psia}$ in the prediction of $P_{T, 2}$.

Some simple uncertainty analyses were performed to see what this level of precision in $P_{T, 2}$ corresponds to in terms of uncertainties in more familiar figures of merit, such as Mach number, dynamic pressure, and normal force coefficient on a representative configuration. The results are presented in Fig. 8, where the uncertainty is given in both engineering units and percentages on the left and right sides of the graphs, respectively. In reviewing these results, the reader should bear in mind that most of the runs made in the facility for research experiments are at stagnation pressures greater than 60 psia. So the large increases in uncertainty seen at the lower stagnation pressures do not apply much of the time. Over the most frequently used range of stagnation pressures, a precision requirement of \pm 0.05 psia for the predicted pitot pressure results in uncertainties of less than $0.4 \%$ in Mach number, $1.5 \%$ in dynamic pressure, and $1.6 \%$ in normal force coefficient for a generic orbiter configuration at $40^{\circ}$ angle of attack. While lower uncertainty is always desirable, it comes at a significantly higher cost in resources. A precision of 0.05 psia in $P_{T, 2}$ was deemed acceptable, and this resulted in a total data volume of 33 points, per Eq. (4).

\section{Locating Points in the Design Space}

Having defined the number of points required to construct a response surface model that will yield predictions to within the specified precision, we now need to decide where those points should be located in the design space. In formal experiment design, points are chosen to allow the response model to have certain properties, such as a minimum overall prediction variance, uniform distribution of the variance over the design space, low correlation between model terms, estimation of the pure error in the experiment, and orthogonal blocking, to name but a few. The distribution of the points in coded design space is what determines these properties. The coded design space is merely the design space where the independent variables are scaled such that -1 and +1 represent the mini-

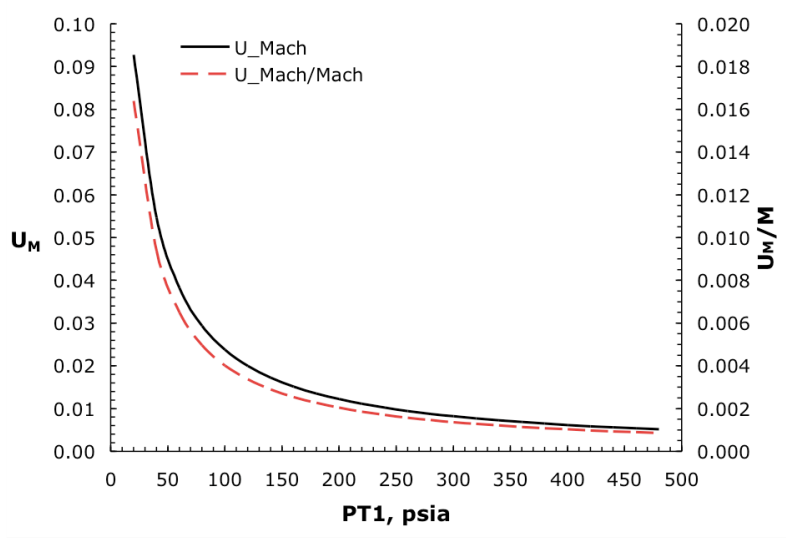

(a) Mach number

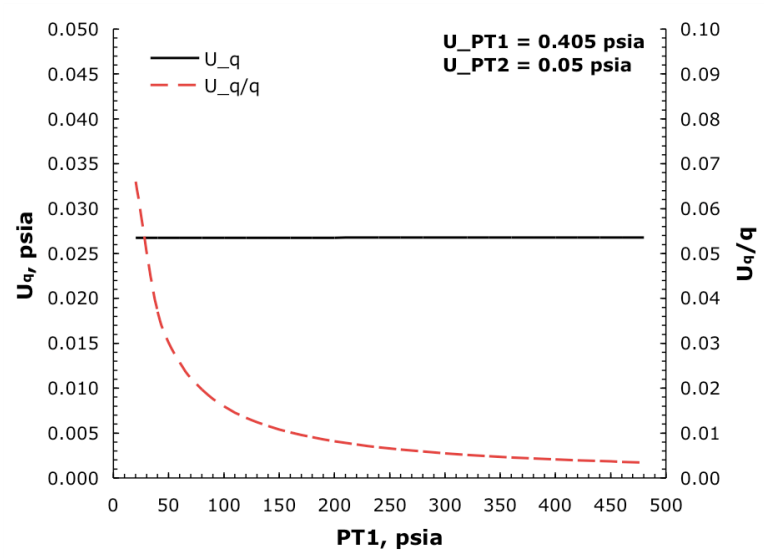

(b) Dynamic pressure

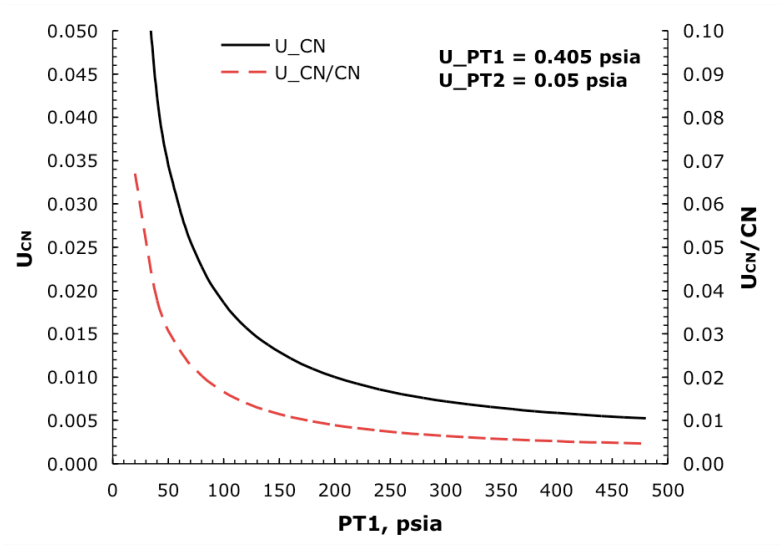

(c) Normal force coefficient, for $\mathrm{C}_{\mathrm{N}}=1.12$

Figure 8. Uncertainties in calculated values given a $\pm \mathbf{0 . 0 5}$ psia precision requirement on pitot pressure. 
mum and maximum values, respectively, over which the response model is to be applied. Hence, a variety of experiment designs can be developed to have specific characteristics, regardless of the magnitudes of the independent variables, for application to a multitude of problems. Some well-known experiment designs for quadratic response surfaces include the central composite, Box-Behnken, and equiradial designs. ${ }^{21}$

For the present calibration experiment, however, the irregular design space resulting from the constraints in the pressure-temperature envelope disallowed the use of such convenient designs. Instead, a custom design was generated using optimal design theory to create what is known as a D-optimal design. A D-optimal design is one that minimizes the volume of the joint confidence region on the vector of regression coefficients, or in other words provides the best balance of minimizing the prediction variance and minimizing the covariance between terms in the response model. ${ }^{22}$ The creation of such designs is always performed on a computer using specific software for the task. In this case, the Design-Expert ${ }^{*}$ software was used for both the generation of the design and the analysis of the experiment.

The locations of the points in the pressure-temperature envelope are shown in Fig.9 (along with the confirmation points, to be discussed in a later section) for the pressure-temperature envelope, and the design points themselves are listed in Table 1. The $D$-optimal design process produced an experiment design totaling 34 points in 23 unique sites with 11 replicated points. Given that only 10 points are needed to fit a quadratic model in three independent variables, the specified design provides 13 degrees of freedom for assessing lack of fit in the response model, and 11 degrees of freedom for computing the pure error. Of particular interest is the pressure-temperature envelope, with constraints in the design space. The $D$-optimal design placed points at the intersections of the boundaries as well as other points along the boundaries and in the interior. The distribution of the points appears "disorderly", but this distribution yields the lowest prediction variance.

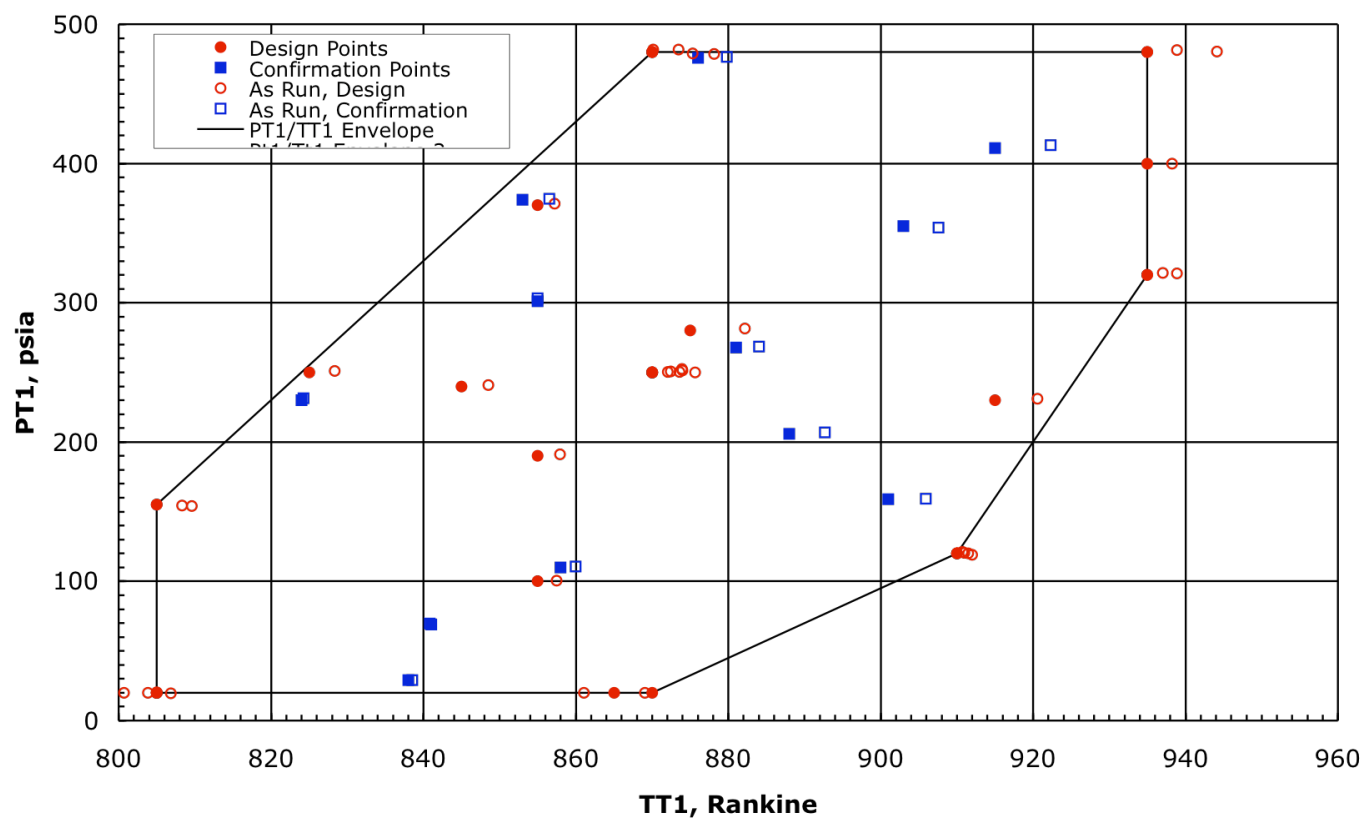

Figure 9. Locations of design and confirmation points in pressure-temperature design space.

After generating an experiment design, several important parameters should be examined to gauge the quality of the design and its ability to accurately predict the response. ${ }^{22}$ Most software packages available for formal experiment design and analysis compute these parameters as a matter of course, making such evaluations relatively straightforward. Usually the first property that is looked at is the scaled prediction variance in the design space. The scaled prediction variance is merely the prediction variance normalized by the intrinsic variability in the measured data, and as such is solely a function of the locations of the design points in the design space. It is desirable to

$\$$ Design-Expert is a registered trademark of Stat-Ease, Inc., Minneapolis, MN. 
have both a low average variance and a relatively uniform and well-behaved distribution of the variance. The prediction variance will always be highest along the boundaries of the design space, but what we want in the interior is a large "flat spot". A plot of the scaled prediction variance (also known as the standard error) for the pressure-temperature space in the calibration experiment is shown in Fig. 10. The prominent "wings" seen in the variance distribution correspond to the constrained regions of the design space where no calibration data was acquired. For the calibration design, the scaled prediction variance ranges from 0.127 to 0.448 , with an average value of 0.294 .

Other important parameters to examine are related to the resolving power of the experiment, the presence of multicollinearity amongst terms in the response model, and the sensitivity of the design to outliers in the measured data. Table 2 lists the power of the experiment design to resolve terms to within two standard deviations of the measurement error, given a 5\% probability for a Type I inference error to occur. The power is the probability that a model term will be rejected when it is indeed false, so high power values are good. For the calibration design, the resolving power for all of the models terms is fairly high, with the exception of the stagnation pressure-temperature interaction term. The much lower value seen here is due to a possible small correlation between pressure and temperature resulting from the constraints in the design space, which will be discussed shortly. Low power values are not necessarily a problem, however, unless the model term one is trying to resolve is very small.

Multicollinearity, or correlation, exists when there are one or more near-linear dependencies among the independent variables in the regression model, and its presence can have a significant effect of the estimates of the model terms and the usefulness of the model in general. The existence of multicollinearity in a design can be detected in a number of ways, two of which are discussed here. The variance inflation factor (VIF) associated with each model term is also listed in Table 2. VIF values of unity indicate no correlation between model terms, and values less than 10 are not troublesome; values greater than 100, however, suggest that correlation is a problem. The highest VIF value seen in the design is for the stagnation pressure-temperature interaction term, although it is

Table 2. Resolving power and variance inflation factors for full quadratic response model.

\begin{tabular}{|c|c|c|}
\hline Model Term & Power at 2 $\sigma(\%)$ & VIF \\
\hline \hline$P T 1$ & 81.1 & 1.70146 \\
\hline$T T 1$ & 70.3 & 2.12983 \\
\hline$X$ & 99.2 & 1.06353 \\
\hline$P T 1^{2}$ & 94.8 & 1.80233 \\
\hline$T T 1^{2}$ & 73.9 & 3.95434 \\
\hline$X^{2}$ & 99.9 & 1.16889 \\
\hline$P T 1 \cdot T T 1$ & 18.6 & 5.31561 \\
\hline$P T 1 \cdot X$ & 81.1 & 1.29344 \\
\hline$T T 1 \cdot X$ & 73.7 & 1.30887 \\
\hline
\end{tabular}

Table 1. Design points for calibration experiment.

\begin{tabular}{|c|c|c|c|c|}
\hline \multirow{2}{*}{ Point } & Pt1 & Tt1 & $\mathbf{X}$ & Phi \\
\cline { 2 - 5 } & \{psia $\}$ & $\{\mathbf{R}\}$ & $\{\mathbf{i n}\}$ & \{deg $\}$ \\
\hline D1 & 480 & 870 & 25 & -45 \\
\hline D2 & 480 & 935 & 13 & 45 \\
\hline D3 & 20 & 805 & 1 & 90 \\
\hline D4 & 280 & 875 & 25 & 90 \\
\hline D5 & 230 & 915 & 13 & -45 \\
\hline D6 & 480 & 870 & 1 & 45 \\
\hline D7 & 20 & 865 & 1 & 0 \\
\hline D8 & 20 & 870 & 13 & 0 \\
\hline D9 & 250 & 870 & 13 & -45 \\
\hline D10 & 20 & 805 & 25 & 0 \\
\hline D11 & 250 & 870 & 25 & 90 \\
\hline D12 & 480 & 870 & 25 & 90 \\
\hline D13 & 320 & 935 & 1 & 45 \\
\hline D14 & 190 & 855 & 13 & 0 \\
\hline D15 & 100 & 855 & 25 & 45 \\
\hline D16 & 250 & 825 & 25 & 90 \\
\hline D17 & 400 & 935 & 25 & 0 \\
\hline D18 & 250 & 870 & 13 & 90 \\
\hline D19 & 120 & 910 & 25 & 0 \\
\hline D20 & 480 & 935 & 13 & 45 \\
\hline D21 & 155 & 805 & 13 & 0 \\
\hline D22 & 250 & 870 & 13 & 45 \\
\hline D23 & 370 & 855 & 13 & 0 \\
\hline D24 & 480 & 870 & 1 & 0 \\
\hline D25 & 120 & 910 & 1 & -45 \\
\hline D26 & 250 & 870 & 1 & 45 \\
\hline D27 & 20 & 805 & 25 & -45 \\
\hline D28 & 240 & 845 & 1 & -45 \\
\hline D29 & 120 & 910 & 25 & -45 \\
\hline D30 & 250 & 870 & 13 & 90 \\
\hline D31 & 20 & 805 & 1 & -45 \\
\hline D32 & 320 & 935 & 1 & 90 \\
\hline D33 & 120 & 910 & 1 & 90 \\
\hline D34 & 155 & 805 & 13 & 45 \\
\hline
\end{tabular}

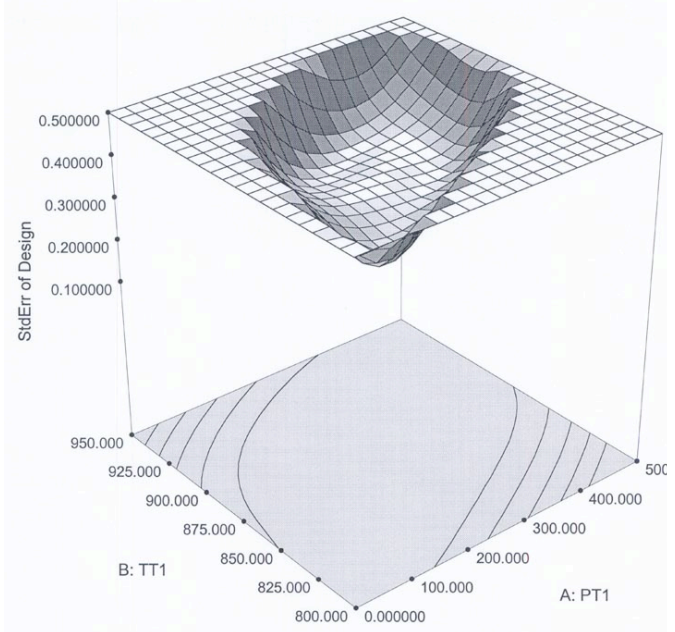

Figure 10. Distribution of scaled prediction variance in design space. 
still well below the problem-threshold value. A look back at the pressure-temperature design space (Fig. 9) shows that because of the operating constraints, two corners of the space are "clipped" and the design points thus fall in a broad diagonal band. If we were to further constrain these corners, the diagonal band in which the points are located would be narrower, and the degree of correlation between pressure and temperature would be greater. In the limit, the points would eventually fall on a straight line, resulting in absolute correlation between pressure and temperature, with no means to separate the independent effects. When using a constrained design space, multicollinearity is something that should be examined carefully. Another means of detecting multicollinearity is to calculate the condition number of the regression matrix as the ratio of the largest to smallest eigenvalues in the matrix. Generally, if the condition number is less than 100 , there is little problem with multicollinearity. For the calibration design, the condition number was 22.06. It should be noted that the VIF and condition number values are given for the case where all of the terms are present in the model, as they should be in the pre-test planning phase. Once the experiment has been run, however, and insignificant terms are eliminated from the response model, small correlations between terms often become "disconnected" and disappear altogether. Hence, the remaining model terms will often have lower VIF values than seen in the pre-test examination of the design matrix.

Finally, the leverage of each design point should be evaluated. The leverage is the ability of a given design point to influence the response model. High leverage points should be avoided since they unduly influence the response model and make it sensitive to outlier points in the data. In general, no one design point should have a leverage value greater than twice the average over all the points in the design space. A high leverage can be reduced by simply replicating the point a number of times in the experiment. The leverage values for the design points in the calibration are plotted in Fig. 11. Results show that all the points have leverage values less than twice the average value, indicating no overly significant influence of any one point.

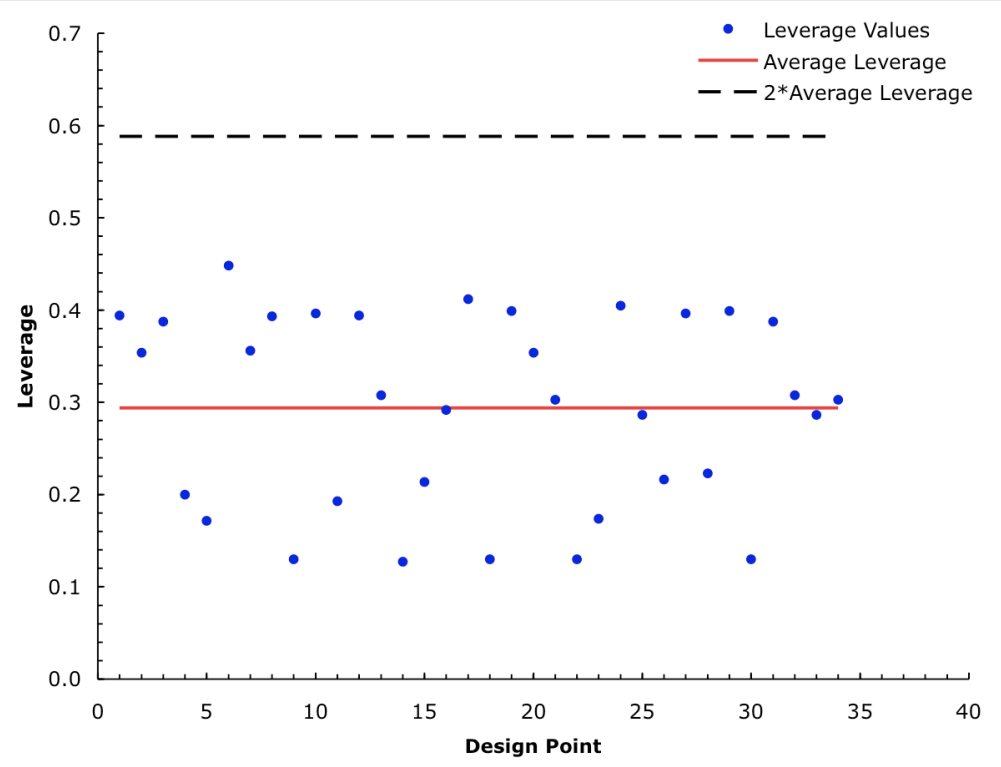

Figure 11. Design point leverage values for calibration experiment.

\section{Confirmation of the Response Model}

A well-designed experiment intended to produce a usable response surface model will generally include multiple degrees of freedom to assess not only the pure error in the experiment but lack of fit in the response model as well. So after all the analysis has been performed, the researcher should have a good idea as to the quality of the response model and its ability to reliably predict the response. In addition to this, however, a response model should be confirmed before it is used. Confirmation usually involves running the experiment at off-design locations in the design space and comparing the measured data with the output of the response model at those locations. The metric by which the comparison is considered "good" or not is if a measured data point falls within the prediction interval of the response model at that location. Recall that the prediction interval is a function not only of the variability in the measured data used to construct the response surface, but also the location within the design space (Fig. 10), and will be highest at the boundaries of the design space. 
As with the design of an experiment, two questions need to be answered regarding the confirmation points: how many points are required, and where should the points be located? The number of confirmation points to use is somewhat subjective, and is a choice based on comfort level, available resources (time and cost), response model complexity, and the criticality of use of the response model. A general rule of thumb would be one-quarter to onethird the number of points used to generate the response model. Not all the confirmation points have to fall within the respective prediction intervals, however, for the model to objectively be considered valid. It is not realistic to require $100 \%$ of the confirmation points to fall within the $95 \%$ prediction interval of the response model. Each measured confirmation point has some degree of experimental error, so a failed confirmation trial does not necessarily reflect on the quality of the model. It is entirely possible for some of the confirmation points to lie outside of the prediction interval due to imperfections in the confirmation data rather than the model. Since a confirmation trial either "passes" or "fails" - a binary solution - confirmation success can be said to follow a binomial probability distribution. As such, the cumulative binomial probability distribution determines the number of successes that can be expected out of a total number of trials, given a probability of success of each trial and the Type I inference risk error probability for the experiment. ${ }^{23}$ This number of expected successes is known as the critical binomial number and is used as an objective criterion for determining whether or not a response model is valid. If the number of successful confirmation trials is greater than or equal to the critical binomial number, than the response model is said to be valid - otherwise it is not.

For the present calibration, we chose to use 12 confirmation points to gauge the adequacy of the response model. Given the 5\% Type I error probability and a $95 \%$ probability of success of an individual confirmation trial, the critical binomial number is 10 . So if at least 10 out of the 12 confirmation points lie within the prediction interval, this test suggests that the model is a good one.

The question as to where the confirmation points should be located is addressed next. One could simply place the points by eye at locations away from the design points. However, unintentionally or otherwise, one may have a predilection to place points more in the interior of the design space, where the model predictions are generally best, or in the corners or on the boundaries where the model performance is weakest. Neither extreme will give a good indication of how well the model represents the data. Therefore, it is best to assign the confirmation points at random. In randomizing the point locations, though, care must be taken to ensure a fair coverage of the design space. It is entirely possible for a small set of confirmation points to cluster in one area of the design space and thus not give an adequate measure of the model. For the calibration experiment, the stagnation pressure range was subdivided into four equal "bins", while the stagnation temperature and axial location ranges were separated into two bins each. The 12 confirmation points were first randomly assigned to the bins such that each bin for a given independent variable contained an equal number of points. Hence, each of the four bins for the stagnation pressure contained three points, while each bin for stagnation temperature and axial location contained six points. Next, the confirmation points were randomly located within each bin. This "binning" process made sure that the entire design space was more or less covered, while the randomization allowed the points to be located without prejudice and with equal probability of landing on or near a boundary or in the interior. The locations of the confirmation points in the design space and relative to the design points are shown in Fig. 9, and the confirmation points are listed in Table 3.

Table 3. Confirmation points for calibration experiment.

\begin{tabular}{|c|c|c|c|c|}
\hline \multirow{2}{*}{ Point } & Pt1 & Tt1 & $\mathbf{X}$ & Phi \\
\cline { 2 - 5 } & $\{$ psia\} & $\{\mathbf{R}\}$ & $\{$ in $\}$ & \{deg $\}$ \\
\hline V1 & 29 & 838 & 17 & -1 \\
\hline V2 & 411 & 915 & 13 & -8 \\
\hline V3 & 206 & 888 & 12 & 81 \\
\hline V4 & 268 & 881 & 3 & 43 \\
\hline V5 & 159 & 901 & 4 & 79 \\
\hline V6 & 301 & 855 & 5 & 21 \\
\hline V7 & 110 & 858 & 1 & 31 \\
\hline V8 & 230 & 824 & 12 & 21 \\
\hline V9 & 476 & 876 & 23 & -11 \\
\hline V10 & 355 & 903 & 25 & -11 \\
\hline V11 & 374 & 853 & 3 & 65 \\
\hline V12 & 69 & 841 & 21 & 57 \\
\hline
\end{tabular}

\section{Execution of the Experiment}

In formally designed experiments, randomization and blocking are two important tactics employed to defend against the effects of persisting time-varying phenomena, known or otherwise, that can occur during a wind tunnel test. $^{24}$ If an experiment is conducted such that the independent variables are changed in some systematic manner, then these time-varying phenomena can cause the response to be biased in a certain direction from the true value, and no amount of replication will remove the bias from the measured results. For this reason, we randomize the order in which the independent variable levels are set to decouple unexplained systematic variations from the true underlying effects. Randomizing an experiment usually results in some decrease in run productivity, and this will need to be accounted for in the planning and design stages. In some experiments, it may be difficult, costly, or impossible to randomize a particular independent variable. For such restrictions in randomization, special experiment 
designs can be used, ${ }^{17}$ although they generally come at the price of increased data volume to achieve an equivalent result.

Blocking refers to the tactic of grouping data points into clusters or "blocks" for analysis. ${ }^{25}$ Data points within a given block tend to have more in common with each other than they do with points in other blocks. For example, in a test lasting a few days, data could be blocked according to the day they were acquired. Or if an instrument were swapped out for another during a test, blocking would be done by which instrument was used. Any systematic variation between blocks is known as a block effect, and if the block effect is large compared to normal chance variations in the data, we say the block effect is significant. Significant block effects can be removed from the unexplained variance to improve precision, and can also provide interesting insights. For instance, if significant block effects were consistently observed between one test day and the next, then it might behoove researchers to investigate the cause.

It is usually advisable to block on factors that may cause some systematic variation, even if none if explicitly anticipated. In the above example, it would be prudent to block the data acquired before and after the replacement of the instrument in order to observe and remove any shift in the data caused by differences in the two instruments. It is often advisable to block on specific time intervals so that the resulting block effects can be used as components of a sum of squares computation to quantify the variance in response model intercept estimates. This provides a direct measure of the bias error due to time-varying sample means caused by persisting changes of the type described above.

The specifics of the test apparatus and instrumentation will be described in detail in the next section. But for the purposes of the following discussion, note that the pitot pressure was measured with a linear array of pressure tubes in a rake mounted to the strut support via a circular sting. As such, only a narrow, one-dimensional strip of the tests section flow was sampled during a given run. The strut is bolted to the arc sector and has no provision for traversing laterally or vertically. To sample different areas of the flow, however, the rake could be rolled manually, between runs, about the sting axis. Early on, rake roll orientation, or azimuthal angle, was considered as an additional independent variable in the experiment. However, Eq. (4) shows that adding roll angle would have increased the required data volume by $50 \%$. Instead, each data point in the design of the three independent variables (stagnation pressure, temperature, and test section axial location) was randomly assigned one of four discrete rake azimuthal orientations: $-45,0,+45$, and $+90^{\circ}$ (Fig. 12). This provided not only temporal randomization to defend against effects persisting over time, as discussed above, but also spatial randomization to average across any azimuthal asymmetry in the facility. As a bonus, this approach permitted azimuthal blocking of the data to determine if there was any systematic variation in the average core flow pitot pressure from one

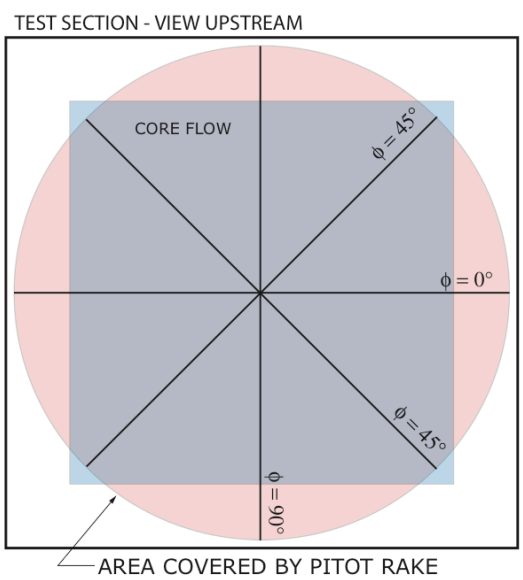

Figure 12. Pitot rake azimuthal orientations and sampled region of test section.

rake orientation to another. The assignments of the rake azimuthal angle were made such that the blocks were approximately equal in size. Nine points each were assigned to the 0 and $90^{\circ}$ rake roll angles, while eight points were allotted to each of the diagonal orientations.

Spatial randomization was also applied to the confirmation points. Instead of being limited to one of four discrete azimuthal orientations, however, the confirmation points were randomly assigned an integer value of roll angle between -45 and $+90^{\circ}$. This allowed confirmation of the model using azimuthal angles other than the ones employed in the construction of the response model.

Rather than executing the series of confirmation points after completing the response model, the confirmation points were run concurrently with the design points, although they were not used in the development of the response model. Both sets of points were grouped together and then randomized as one unit, so that the confirmation points are randomly sown in amongst the design points.

\section{Test Apparatus and Experimental Procedure}

The hardware and instrumentation used in the calibration experiment are described in this section along with the procedures followed in executing the experiment and analyzing the data for each run. 


\section{A. Pitot Pressure Measurements}

The pitot pressure, $P_{T, 2}$, was measured using a rake with a linear array of 151 pressure tubes spaced 0.125 inches between centers, spanning a distance across the test section of \pm 9.375 inches from the centerline. The individual probes were comprised of stainless steel tubing $0.035 / 0.020$ inches (outer/inner) diameter, with the probes extending 1.000 inches in front of the body of the rake. Inside the body of the rake, the tubing diameter was increased to $0.060 / 0.035$ inches diameter, and the total tubing length was approximately 8 feet. At the other end, the tubing was brazed onto head plates for the ESP pressure transducer modules. This method of attachment minimized the number of connections in the tubing path, reducing the chance for leaks. The rake was permanently fixed to a 1-inchdiameter sting of 9 inches in length. A photograph of the pitot rake installed in the test section of the 20Inch Mach 6 Tunnel is shown in Fig. 13.

The pitot rake was originally built for the calibration of a new axisymmetric nozzle for the Langley 20Inch $\mathrm{CF}_{4}$ Tunnel, a hypersonic facility that utilizes a heavy gas to simulate aspects of a real-gas flow. ${ }^{26}$ The fine spacing of the pitot probes was needed to locate small disturbances that could focus on the centerline of that facility, and such spacing required the use of small diameter probes. Before using the rake in the present calibration, however, it was necessary to make sure the tight spacing would not result in mutual interference between neighboring probes, or that the measured pressures would be influenced by viscous effects in the small tubes. A series of inviscid Euler solutions were run using the unstructured FELISA $\operatorname{code}^{27}$ on a single pitot tube and two neighboring tubes of varying spacing. The post-normal-shock stagnation pressures for the twin-tube cases were compared with that of a single pitot tube as an indica-

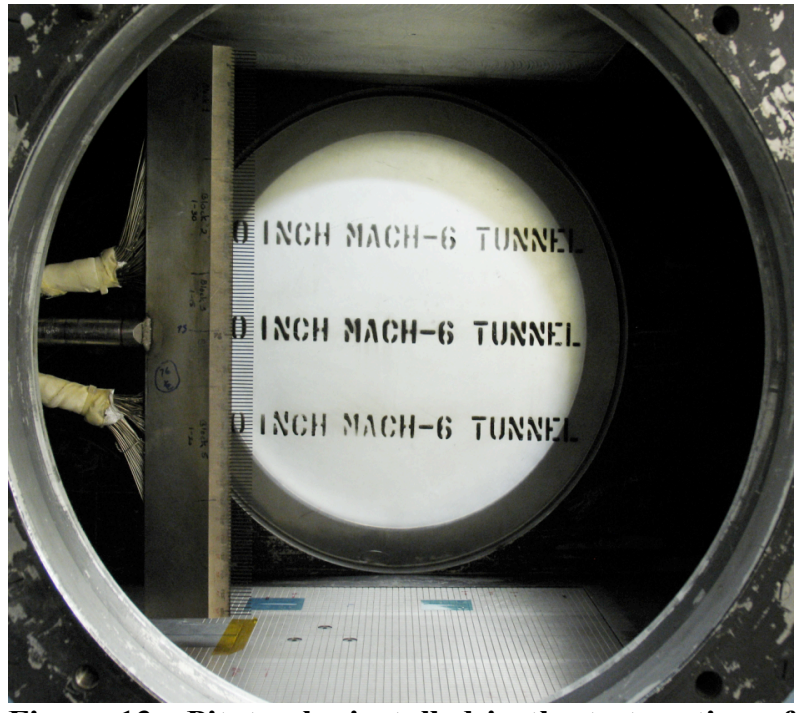

Figure 13. Pitot rake installed in the test section of the 20-Inch Mach 6 Tunnel.

tion of any mutual interference. Results show that the spacing between tube centers must be within 3 pitot tube diameters for any interference to occur. The spacing on the present rake is 3.57 tube diameters, suggesting no interference problems. It is well known that viscous effects can bias pressure measurements when small diameter probes are used at low Reynolds numbers. The ratio of the measured pressure to the true value has been correlated through numerous experiments as a function of product of the post-normal-shock Reynolds number (based on probe outer radius $^{\S \S}$ ) and the shock density ratio. ${ }^{28,29}$

$$
\frac{P_{T_{2, \text { measured }}}}{P_{T_{2, \text { true }}}}=f\left(\operatorname{Re}_{2} \cdot \sqrt{\rho_{2} / \rho_{\infty}}\right)
$$

Empirical findings have shown that for air and nitrogen, no bias in the pressure measurements occurs for values of the correlation parameter greater than 200 for a cold-wall condition and 500 for an adiabatic-wall condition. For the present calibration experiment, the correlation parameter ranges from 213 to over 46,000 . Given the low thermal mass of the probes, the probe wall temperature is probably closer to the adiabatic limit, indicating some viscous influence on the pressure measurements at the lowest stagnation pressures. However, the bias is less than $0.5 \%$, within the measurement precision of the ESP transducers at these low pitot pressures.

Five 32-port, 15-psid ESP modules were used for the pitot pressure measurements. Sequential groups of approximately 30 tubes were assigned to each module, and four pitot tubes (out of the 151 total) were teed into two adjacent modules to identify any module-to-module differences. The pressure measurements were made with the reference side of the transducers pulled down to a hard vacuum with a combination of oil diffusion and mechanical turbo-pumps, and the reference pressure was measured with a high-accuracy Hastings gauge. The ESP modules were attached to a heater base and installed in a protective enclosure on the arc sector and beneath the floor plate. A controller on the heater base maintained the ESP modules at a steady temperature of $115^{\circ} \mathrm{F}$, as measured by a Type E thermocouple affixed to the exterior of one of the modules.

$\S \S$ With the implicit assumption that the ratio of the inner to outer radii is large enough to avoid orifice effects. 
Immediately prior to each run, an in-situ calibration of the ESP transducers was performed using the built-in capability of the System 8400. A series of five user-selected pressures was set, spanning the range of the transducers, and measured with a high-accuracy standard. The voltage responses of all the individual ESP transducers were recorded and curve-fit to the measured pressures. The same in-situ calibration was conducted immediately after a run in order to ascertain any changes that may have occurred due to environmental effects. In both instances, the ESP calibrations were done with the test section under a vacuum for consistency. Unfortunately, the System 8400 is hard-wired to return a fourth-order polynomial fit to the five set calibration pressures, leaving no residual degrees of freedom for determining the quality of the fit. The individual transducers have a near-linear voltage response with pressure, so no more than a quadratic fit should be needed. Therefore, for each transducer, the pre and post-run calibrations were combined, and a quadratic fit to the net 10 points was generated, leaving 2 lack-of-fit and 5 pure error degrees of freedom for computing the regression standard errors of each individual transducer.

In the 20-Inch Mach 6 Tunnel, the strut is bolted to a narrow pad on the arc sector. The spacing of the fastener holes allows placement of the strut over an 8-inch range of the test section axial location in 2-inch increments. For the calibration experiment, the strut was set in the rearward-most position, and the axial location of the pitot rake was adjusted using two separate sting adaptors. Roll orientation was set using an inclinometer to measure the angle. The pressure tubing bundles were wrapped in insulating cloth and tape to reduce heating to the tubes, and were tied down to the sting and strut to keep the tubing out of the flow as much as possible. Adjusting the roll orientation of the rake was straightforward and accomplished fairly easily. Changing the rake axial location, however, was a timeconsuming process that took longer than expected. In planning for an experiment that will be run in a randomized sequence, careful thought should be given to the selection of hardware for ease of change. The pitot rake used in the present calibration was existing hardware available at the time, but it was not designed for easy manipulation. Future calibrations will use purpose-built hardware to better realize the resource savings possible with designed experiments.

\section{B. Stagnation Pressure and Temperature Measurements}

The stagnation pressure in the settling chamber of the wind tunnel was measured using two absolute Druck transducers with pressure ranges of 0-150 and 0-500 psia, and calibrated uncertainties of \pm 0.057 and \pm 0.405 psia, respectively, at a $95 \%$ confidence level. Although the lower range transducer could yield more accurate measurements at low pressures, the stagnation pressure was read from the higher capacity gauge throughout the experiment to avoid any gauge-to-gauge bias effects.

Stagnation temperature was measured with a pair of shielded Type $\mathrm{J}$ thermocouple probes in the settling chamber, each with a stated uncertainty of $\pm 3 \mathrm{R}$. The stagnation temperature was taken as the average of the two readings, with an uncertainty on the average value of $\pm 2.12 \mathrm{R}$. Five external resistance temperature detectors (RTD) provide a reference temperature for the thermocouples.

\section{Wall Static Pressure Measurements}

The optical window on top of the test section was replaced with a metal blank containing four static pressure ports for measuring wall pressures on the upper centerline at axial locations of $X=1.10,7.06,14.15$, and 20.45 inches. The pressures were measured using individual variable-capacitance, diaphragm-type gauges from Datametrics with a pressure range of $0-1000$ Torr. Uncertainty at a $95 \%$ confidence level is stated as $\pm 0.07 \%$ of reading $\pm 0.01 \%$ of the full-scale range. The transducers were mounted exterior to the test section on heater bases directly on top of the metal blank. The heater bases maintained the gauges at a controlled, steady temperature of $105^{\circ} \mathrm{F}$, while the close proximity of the instruments to the pressure ports resulted in short tubing lengths that minimized pressure lag in the measurements.

\section{Experimental Procedure}

Because of the randomized order of the experiment, only one design point was accomplished per tunnel run. Prior to a run, the axial position and/or roll orientation of the pitot rake were set, with pressure tubing bundles rewrapped, adjusted, and restrained as necessary (the time-consuming task mentioned above). The tunnel was started, the stagnation pressure and temperature were brought to the desired conditions, and the rake was injected into the flow from its sheltered position below the test section. A continuous stream of data was recorded for 30 seconds at a rate of 20 samples/sec and filtered using a 1-Hz low-pass filter. At the end of this data-recording period, the rake was retracted from the flow prior to flow stoppage. Flow conditions were reduced using the GASPROPS code ${ }^{30}$, a computational algorithm utilizing a multi-coefficient virial gas equation of state to determine the flow properties of an arbitrary real gas. 


\section{E. Data Reduction}

Following a run, the data were processed and analyzed. Time history plots were made of the stagnation pressure and temperature, the four wall pressures, and the centerline pitot probe on the rake. A representative plot is shown in Fig. 14 for Design Point \#30, where the nominal stagnation pressure and temperature were 250 psia and 870 R, respectively, and the axial location was set at $X=13$ inches. From Fig. 14, a pressure-temperature combination was chosen closest to the design values. An exact match was seldom achieved, due to difficulties in maintaining the operating conditions so precisely, especially with temperature. Preference was given toward choosing a closer pressure value since it was known that the pitot pressure was a much stronger function of stagnation pressure than temperature. The small deviations from the design levels will result in a slight change in the distribution and average value of the scaled prediction variance of the design since the points are not exactly at the "optimum" locations specified by the $D$-optimal design. It is important, though, to use the as-measured values in the creation of the response model instead of the nominal ones.

For Design Point \#30, the closest pressure-temperature match to the nominal conditions occurred at approximately $t=4$ seconds, and a check of the rake centerline pitot pressure reveals no pressure lag at that time. The pitot pressure distribution across the test section was plotted, as illustrated in Fig. 15, and an average pitot pressure value was calculated over a span chosen as the core flow - in this case, \pm 6.75 inches. Outlier points, most likely due to leaks in pressure tubes, were identified and omitted from the averaging. This average pitot pressure value was recorded as the response variable of interest in the experiment.

\section{Results and Discussion}

In the following section, the construction and validation of the response model are discussed, along with the predicted trends. Various blocking schemes are presented, and comparisons with previous calibration results are made.

Before getting to the specific results, a brief explanation of the analysis of variance (ANOVA) technique is given to set the stage for the discussions to follow. An in-depth discussion of ANOVA is well beyond the scope of this paper, and the reader is referred to the references for further details. In short, ANOVA is a method used to test for significance of regression, and is based on the decomposition of the total variability in the response. ${ }^{31}$ That is, the net change seen in the response variable can be broken down into separate components due to individual model terms, blocks, and the residual pure error and lack-of-fit error. A measure of the variability of a model term is contrasted with the total error to see if the term is statistically significant as compared to the error. This can be thought of as a signal-to-noise ratio. The use of ANOVA provides an objective means of discriminating the significant terms in the response model according to tolerances set by the user. Modern software packages make ANOVA a speedy and routine process.

\section{A. Development of the Response Model}

The calibration experiment was performed in the randomized order described previously, and the average pitot pressures across the core flow of the test section were recorded as the response variable. The as-run stagnation pressures and temperatures for both the design and validation points are shown in Fig. 9

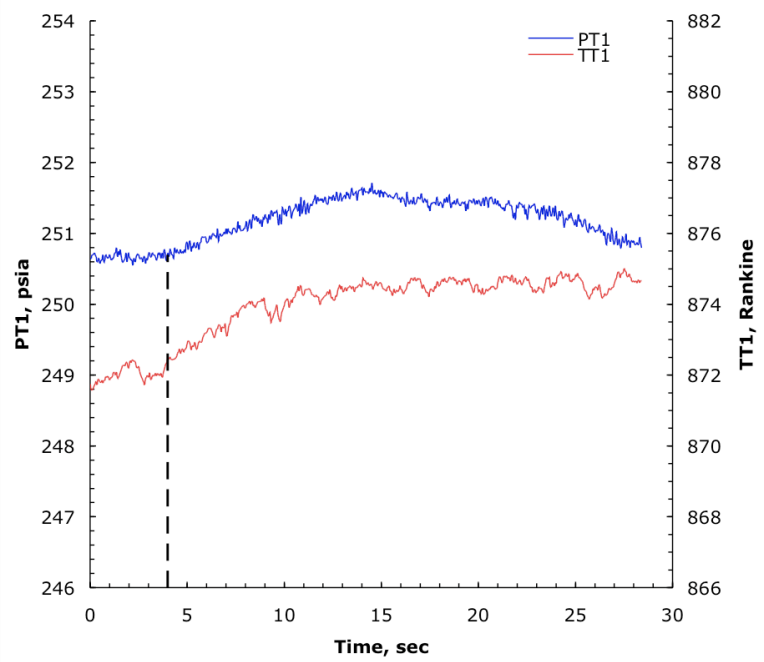

Figure 14. Time histories of stagnation pressure and temperature for Design Point \#30.

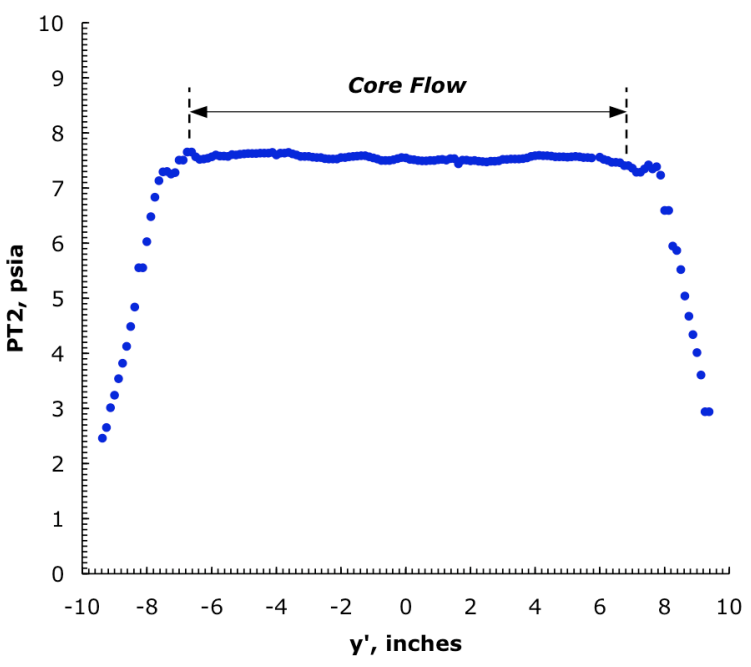

Figure 15. Variation of pitot pressure along rake for Design Point \#30 at $t=4$ sec.

18

American Institute of Aeronautics and Astronautics 
in comparison with the nominal values specified in the design. In general, temperature deviated more from the design values than pressure since the thermal mass of the wind tunnel made precise setting of the temperature difficult, and, as mentioned previously, preference was given towards pressure when selecting the operating conditions from the time-history data.

The results of an ANOVA test on the unblocked data are shown in Table 4 for the full quadratic model. Some explanation of the ANOVA table is given here to orient the reader to the meaning of the results. The sum of squares is a measure of how much change in the pitot pressure is due to each term in the model and the residual error and its components. It is easily seen that the stagnation pressure is the dominant effect, as expected, providing $99.95 \%$ of the variability in the model. The mean square is computed as the sum of squares divided by the corresponding degrees of freedom, and represents the average effect on the response from each degree of freedom. The mean square of the residual is an unbiased estimate of the uncertainty in the model, so a ratio of the mean square of a model term to the mean square of the residual - the F-value - provides a measure of the significance of that model term. The last column gives the probability that the associated F-value could occur due to noise.

Since the Type I inference error probability was specified at $5 \%$, we reject any model term in the ANOVA table where Prob $>\mathrm{F}$ is greater than 0.05. As discussed earlier, we want to avoid accepting erroneous model terms, as this increases the uncertainty in the model. Using this criterion, the $P T 1^{2}, T T 1^{2}$, and $T T 1 \cdot X$ terms are excluded from the model. Figure 16 provides a clear illustration of why certain terms were kept and others rejected. A unit normal probability density function shows each of the model

Table 4. ANOVA results for unblocked, full quadratic model.

\begin{tabular}{|c|c|c|c|c|c|}
\hline Source & $\begin{array}{l}\text { Sum of } \\
\text { Squares }\end{array}$ & $\begin{array}{c}\text { Degrees of } \\
\text { Freedom }\end{array}$ & Mean Square & F-Value & Prob $>$ F \\
\hline Model & 402.679 & 9 & 44.7422 & 17990.0 & $<0.0001$ \\
\hline PT1 & 402.482 & 1 & 402.4820 & 161831 & $<0.0001$ \\
\hline TT1 & 0.0486688 & 1 & 0.0486688 & 19.5689 & 0.000180 \\
\hline$x$ & 0.0840057 & 1 & 0.0840057 & 33.7772 & $<0.0001$ \\
\hline PT1 ${ }^{2}$ & $1.9526 \mathrm{E}-06$ & 1 & $1.9526 \mathrm{E}-06$ & $7.8509 E-04$ & 0.977878 \\
\hline$T T 1^{2}$ & 0.0036411 & 1 & 0.0036411 & 1.46402 & 0.238074 \\
\hline$x^{2}$ & 0.0172243 & 1 & 0.0172243 & 6.92559 & 0.014618 \\
\hline PT1-TT1 & 0.0246037 & 1 & 0.0246037 & 9.89272 & 0.004383 \\
\hline$P T 1 \cdot X$ & 0.0192148 & 1 & 0.0192148 & 7.72594 & 0.010411 \\
\hline$T T 1 \cdot X$ & $1.2776 \mathrm{E}-05$ & 1 & $1.2776 \mathrm{E}-05$ & $5.1368 \mathrm{E}-03$ & 0.943457 \\
\hline Residual & 0.0596892 & 24 & 0.0024871 & & \\
\hline Lack of Fit & 0.0350879 & 13 & 0.0026991 & 1.20684 & 0.381906 \\
\hline Pure Error & 0.0246013 & 11 & 0.0022365 & & \\
\hline Total & 402.739 & 33 & & & \\
\hline
\end{tabular}

term coefficients as a multiple of the standard error in estimating it. The rejected terms are well inside the normal distribution, indicating that their corresponding regression coefficients cannot be distinguished from zero with at least $95 \%$ confidence.

The resulting model is known as a reduced quadratic model and has the following form:

$$
P_{T_{2}}=b_{0}+b_{1} P_{T_{1}}+b_{2} T_{T_{1}}+b_{3} X+b_{4} P_{T_{1}} T_{T_{1}}+b_{5} P_{T_{1}} X+b_{9} X^{2}
$$

After model terms are removed, a new ANOVA test must be performed since we are now fitting a different model to the data. The ANOVA results for the unblocked, reduced quadratic model are shown in Table 5. The number of error degrees of freedom increased by 3 since the rejected model terms and their effect on the response are now counted towards the lack of fit error. In the new model, the mean square of the residual is slightly less, resulting in an improved ability to distinguish small effects, although there is no change in which terms remain in the model. Lack of fit is not significant, as given by the high probability value.

Several parameters can be calculated as measures of the quality of the response model, and are also given at the bottom of Table 5. The standard

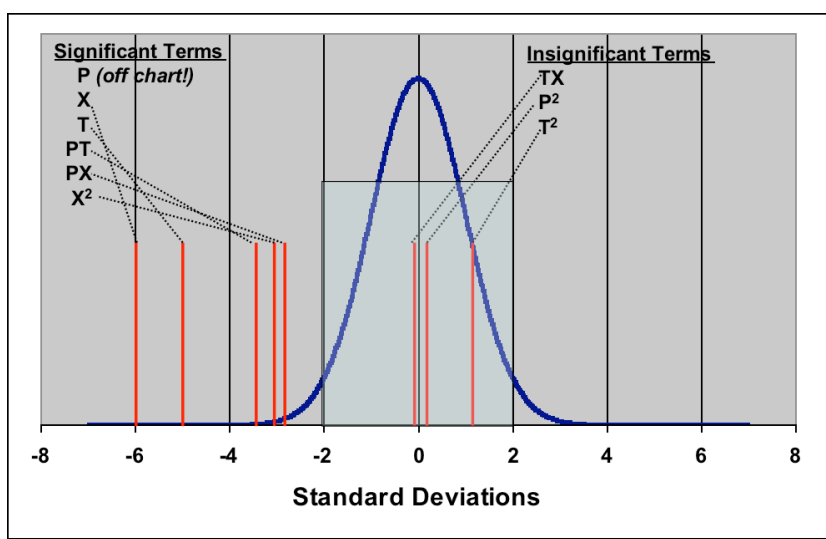

Figure 16. Significance of model terms relative to the standard error.

deviation, $\sigma$, is listed as well as the familiar $R^{2}$ value, here indicating a very good fit of the model to the data. But a high $R^{2}$ value alone is not the best indicator of a good model. The $R^{2}$ value merely says how well the model "goes 
through the data points." Adding model terms, including insignificant ones, will always increase $R^{2}$ even while degrading the model and increasing the uncertainty. For this reason, the adjusted $R^{2}$ is a better indicator since it takes into account the number of model terms, and will actually decrease if negligible terms are added. The predicted $R^{2}$ and PRESS (Prediction Error Sum of Squares) values are both measures of how well the model will predict the response. Small values of PRESS are desired. Adequacy of precision is a signal-to-noise ratio for the model as a whole, and indicates an ability to use the model to navigate the design space. Values greater than 4 indicate reasonable prediction performance. For the present case, all indicators point to a well-constructed response model that should yield excellent predictions of the pitot pressure over the calibrated ranges of stagnation pressure, temperature, and axial location within the test section.

\section{B. Analysis of Residuals}

Before the newly constructed response model can be accepted, we must first make sure that certain assumptions inherent in the use of the ANOVA technique hold true. The validity of the results from ANOVA are predicated on the requirement that the residuals (the differences between measured values and predictions from the model at the design points) are normally and independently distributed, with zero mean and a variance $\sigma^{2}-$ commonly abbreviated as $\operatorname{NID}\left(0, \sigma^{2}\right) .{ }^{17}$ That is, the residuals follow a normal probability distribution, are independent of the order in which the data were acquired, and have Table 5. ANOVA results for unblocked, reduced quadratic model.

\begin{tabular}{|c|r|c|r|r|r|}
\hline Source & $\begin{array}{c}\text { Sum of } \\
\text { Squares }\end{array}$ & $\begin{array}{c}\text { Degrees of } \\
\text { Freedom }\end{array}$ & Mean Square & \multicolumn{1}{c|}{ F-Value } & \multicolumn{1}{c|}{ Prob > F } \\
\hline \hline Model & 488.609 & 6 & 81.4349 & 34328.6 & $<0.0001$ \\
\hline $\boldsymbol{P T 1}$ & 488.365 & 1 & 488.3650 & 205868 & $<0.0001$ \\
\hline $\boldsymbol{T T 1}$ & 0.0494607 & 1 & 0.0494607 & 20.8500 & $<0.0001$ \\
\hline $\boldsymbol{X}$ & 0.0882338 & 1 & 0.0882338 & 37.1946 & $<0.0001$ \\
\hline $\boldsymbol{X}^{\mathbf{2}}$ & 0.0183857 & 1 & 0.0183857 & 7.75042 & 0.009689 \\
\hline $\boldsymbol{P T 1} \cdot \boldsymbol{T T 1}$ & 0.0623888 & 1 & 0.0623888 & 26.29977 & $<0.0001$ \\
\hline $\boldsymbol{P T 1} \cdot \boldsymbol{X}$ & 0.0260009 & 1 & 0.0260009 & 10.96058 & 0.002648 \\
\hline \hline Residual & 0.0640499 & 27 & 0.0023722 & & \\
\hline Lack of Fit & 0.0394486 & 16 & 0.0024655 & 1.10242 & 0.445250 \\
\hline Pure Error & 0.0246013 & 11 & 0.0022365 & & \\
\hline \hline Total & 488.674 & 33 & \multicolumn{3}{|l}{} \\
\hline
\end{tabular}

\begin{tabular}{|c|r||c|r|}
\hline $\boldsymbol{\sigma}$ & 0.0487054 & $\mathbf{R}^{\mathbf{2}}$ & 0.999905 \\
\hline PRESS & 0.0995117 & Adjusted R $^{\mathbf{2}}$ & 0.999884 \\
\hline Adeq. Prec. & 621.732 & Predicted R $^{\mathbf{2}}$ & 0.999853 \\
\hline
\end{tabular}

an average value close to zero while yielding a variance of the model that is independent of the location within the design space. Gross violations of these assumptions can significantly affect the coefficient estimates in the model. Fortunately, the correctness of the assumptions may be quickly revealed with some simple plots. As an example, a normal probability plot of the residuals is presented in Fig. 17. Points that are normally distributed fall on a straight line when plotted on a probability scale, while points off the line can be assumed to have some systematic error component that may be attributable to lack of fit errors in the model. Although not every point is riveted to a precisely straight line, it is clear that the residuals lie sufficiently close to a straight line on the probability scale to be described as normally distributed. ANOVA is fairly robust to deviations from the normality assumption, so that the technique can still be used even when moderately skewed probability distributions are present.

The independence assumption is checked by plotting the residuals versus time, or the order in which the points were acquired. A pattern to the residuals indicates that some correlation exists between the points, which can bias the estimates of the model coefficients. Violations of this assumption can be difficult to correct for after the fact, so it is important to ensure independence in the execution of the experiment, mostly through proper randomization.

Likewise, potential non-constant variance can be identified via plots of the residuals versus the independent variables and the predicted response values. Lack of homogeneity in the variance can skew the results of an $F$-test, affecting the development of the response model. Such non-constant variance can be accounted for through the use of variance-stabilizing transformations, where the response variable is transformed via a square root or logarithmic function, say, and ANOVA is run on the transformed data. All told, the analysis of the residuals of the calibration experiment uncovers no evidence of violations of the

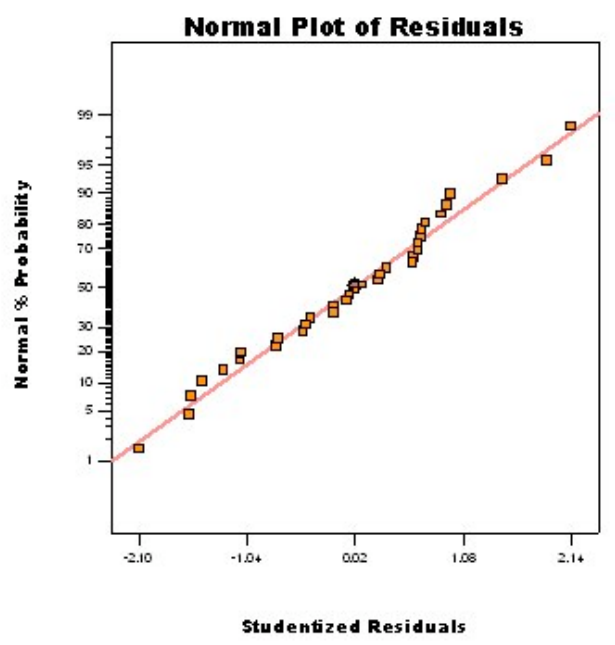

Figure 17. Normal probability plot of the residuals from the reduced quadratic response model. 
assumptions implicit in the use of ANOVA.

\section{Confirmation of the Response Model}

A response model should be confirmed before it is used to make predictions of the behavior of a system. As discussed earlier, 12 random confirmation points were acquired during the calibration experiment. These points were not used in the development of the response model, but rather set aside for later comparisons with predictions. A confirmation trial is deemed successful if the measured datum falls within the $95 \%$ prediction interval of the response model. Figure 18 shows the difference between measured value and prediction for the 12 confirmation points, along with the $95 \%$ prediction interval for the model. Note that the prediction interval is a function of the location in the design space and thus is not a constant value. All twelve of the confirmation points fall within the prediction interval, tentatively indicating that we have an adequate model.

Bear in mind, however, that any confirmation trial can be made successful if the $95 \%$ prediction interval is wide enough. It is necessary but not sufficient for a certain number of confirmation trials to be successful; the precision specified in the pre-test requirements must also be met. To this end, we compute the $95 \%$ confidence intervals about the predictions that represent the ranges in which the true response is expected to fall. These confidence intervals are shown in Fig. 19 to lie within the pre-test requirements, indicating that the precision requirements were achieved.

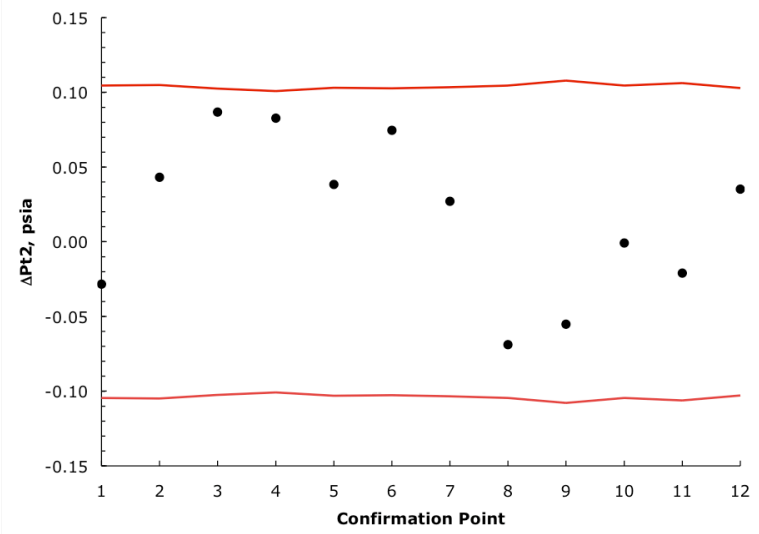

Figure 18. Differences between confirmation results and response model predictions as compared to prediction intervals.

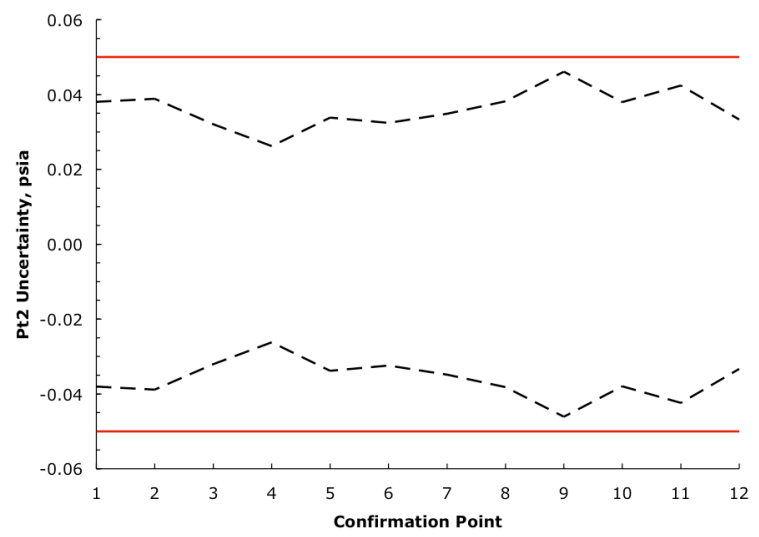

Figure 19. Comparison of prediction confidence intervals with pre-test requirements.

\section{Block Effects}

The use of blocking was previously identified as a means to detect an effect of the pitot rake azimuthal orientation. The design was blocked according to the roll angle, and a new ANOVA test was performed. A block effect reveals itself as a shift in the intercept of the response model, with each block contributing a different bias. We deem a block effect to be significant if the shifts due to the blocks are greater than some figure of merit, in this case the average prediction interval for the response model, given as:

$$
\overline{P I}=2 \sqrt{\frac{p \sigma^{2}}{N}}
$$

Table 6 shows the block effects for the design blocked according to rake orientation angle, along with the resulting standard deviation from the new ANOVA test. For $p=7$ model terms (Eq.(6)) and $N=34$ design points, the average prediction interval is \pm 0.03945 psia. Since the block effects fall within this interval, we are unable to conclude that any significant effect of rake orientation angle was present. The data were also blocked on time intervals into 2, 3, and 4 blocks. Al-
Table 6. Block effects for rake orientation.

\begin{tabular}{|c|r|}
\hline Block & Effect, psia \\
\hline \hline$\phi=-45^{\circ}$ & 0.0320131 \\
\hline$\phi=0^{\circ}$ & -0.0306928 \\
\hline$\phi=45^{\circ}$ & 0.0114123 \\
\hline$\phi=90^{\circ}$ & -0.0127326 \\
\hline \multicolumn{2}{|c|}{$\sigma=0.04347$ psia } \\
\hline
\end{tabular}


though not shown here, results indicated no systematic changes in the data with time.

\section{E. Trends in Pitot Pressure From the Response Model}

Having developed a response model for pitot pressure and confirmed its adequacy, we can now use the model to investigate trends in the response and make predictions of pitot pressure over the range of independent variables in the design space. The coefficient estimates from ANOVA for a response model with independent variables in coded units are given in Table 7, along with the 95\% confidence intervals and VIF values for each term. Recall that in coded units, each independent variable ranges from -1 to +1 , thus allowing a comparison of the magnitudes of the coefficients of each term as a measure of their effect on the response. As expected, the stagnation pressure, $P_{T, 1}$, is by far the dominant term. The coefficient magnitudes relative to $P_{T, I}$ are plotted in Fig. 20. Note the use of a logarithmic scale to allow the lesser terms to be seen. Excepting the pressure-temperature interaction, all the other terms in the model have less than $1 / 100^{\text {th }}$ of the effect of the stagnation pressure. The VIF values all indicate that there are no correlations between terms in the model.

To look at the direction in which a model term affects the response, however, we must use the response model with independent variables given in engineering units and inspect the signs of the coefficients, given in Table 8. Pitot pressure, of course, increases with stagnation pressure, and in a linear sense as expected from previous calibration trends, as illustrated in Fig. 21 for the two extremes in stagnation temperature. Increasing stagnation temperature also results in an increase in pitot pressure, which for a fixed stagnation pressure means a reduction in Mach number. This is likely due to the decrease in unit Reynolds number with increasing temperature, thickening the wall boundary layer and lowering the effective area ratio of the nozzle. However, the stagnation pressure-temperature interaction term acts in a negative sense, causing a net reduction in $P_{T, 2}$ for increasing temperatures at stagnation pressures greater than 89 psia. The exact physical mechanism in effect is unknown at this time, but a few reasons are postulated here. When stagnation temperature is held constant, the Reynolds number increases with stagnation pressure, resulting in a thinner boundary layer and a corresponding effective increase in the nozzle area ratio; hence, a larger Mach number and lower pitot pressure. Likewise, for pressures greater than 89 psia,

Table 7. Coefficient estimates from ANOVA for response model with variables in coded units.

\begin{tabular}{|c|r|c|r|r|r|}
\hline Model Term & $\begin{array}{c}\text { Coefficient } \\
\text { Estimate }\end{array}$ & $\begin{array}{c}\text { Degrees of } \\
\text { Freedom }\end{array}$ & 95\% CI Low & 95\% CI High & \multicolumn{1}{c|}{ VIF } \\
\hline \hline Intercept & 7.51906 & 1 & 7.48833 & 7.54979 & \\
\hline $\boldsymbol{P T 1}$ & 6.76399 & 1 & 6.73340 & 6.79458 & 1.39844 \\
\hline $\boldsymbol{T T 1}$ & -0.0713668 & 1 & -0.1034360 & -0.0392978 & 1.48203 \\
\hline $\boldsymbol{X}$ & -0.0645587 & 1 & -0.0862785 & -0.0428389 & 1.03920 \\
\hline $\boldsymbol{X}^{\mathbf{2}}$ & -0.0489982 & 1 & -0.0851108 & -0.0128856 & 1.01392 \\
\hline $\boldsymbol{P T 1} \cdot \boldsymbol{T T 1}$ & -0.1020120 & 1 & -0.1428270 & -0.0611976 & 1.084530 \\
\hline $\boldsymbol{P T 1} \cdot \boldsymbol{X}$ & -0.0485400 & 1 & -0.0786233 & -0.0184568 & 1.027370 \\
\hline
\end{tabular}

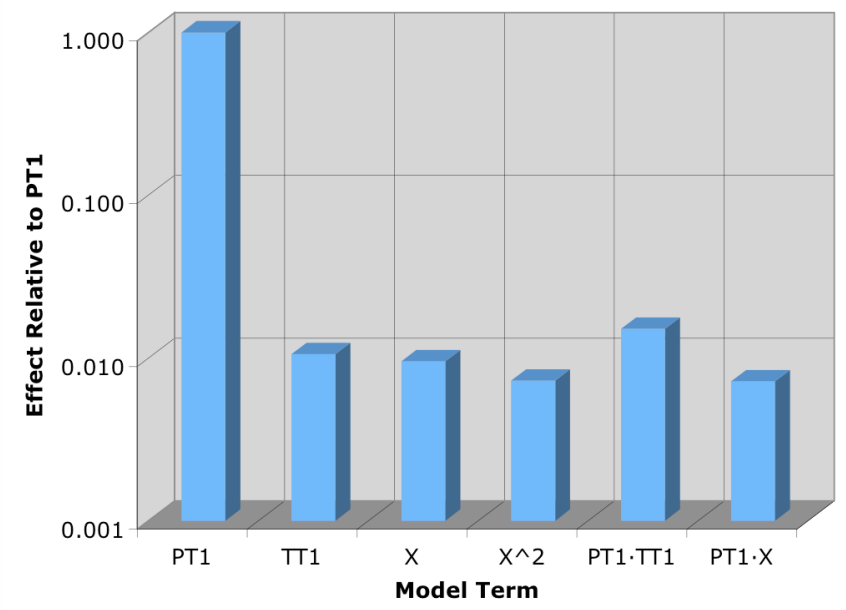

Figure 20. Relative effects of response model terms on the pitot pressure.

increasing temperature creates a net decrease in $P_{T, 2}$, perhaps through an effect of the wall-temperature ratio, $T_{w} / T_{T, I}$. It is well known that lowering the wall-temperature ratio (i.e. raising $T_{T, 1}$ ) results in a thinner boundary layer ${ }^{32}$, and

Table 8. Coefficient estimates from ANOVA for reduced quadratic response model.

\begin{tabular}{|c|c|}
\hline Model Term & $\begin{array}{c}\text { Coefficient } \\
\text { Estimate }\end{array}$ \\
\hline \hline Intercept & $-4.06742 \mathrm{E}-01$ \\
\hline PT1 & $3.55738 \mathrm{E}-02$ \\
\hline TT1 & $6.07944 \mathrm{E}-04$ \\
\hline $\boldsymbol{X}$ & $7.86375 \mathrm{E}-03$ \\
\hline $\boldsymbol{X}^{\mathbf{2}}$ & $-3.40265 \mathrm{E}-04$ \\
\hline PT1·TT1 & $-6.82358 \mathrm{E}-06$ \\
\hline PT1 $\boldsymbol{X}$ & $-1.75870 \mathrm{E}-05$ \\
\hline
\end{tabular}

wall-temperature ratio can also have an effect on boundary layer transition. The third reason hypothesized has nothing to do with any fluid dynamic phenomena, but rather the design of the facility itself. The 20-Inch Mach 6 Tunnel utilizes an un-cooled nozzle, so thermal expansion of the nozzle, particularly at the throat where the heat flux is greatest, may cause a small change in the area ratio. The amount of thermal expansion would be a function of the mass flow rate, with greater convective heat transfer to the throat at high stagnation pressures.

Pre-test analysis of previous calibration results revealed a quadratic effect of test section axial location on the pitot

American Institute of Aeronautics and Astronautics 
pressure, and this effect - the axial Mach number gradient - was indeed seen in the present data. Figure 22 illustrates the change in pitot pressure with axial distance relative to the pitot pressure at the forward-most calibrated location in the test section for several values of stagnation pressure. At lower stagnation pressures, the pitot pressure increases for some distance downstream before falling again. The peak magnitude of this increase diminishes and moves upstream as the stagnation pressure becomes larger, disappearing altogether at some pressure greater than 300 psia. In most hypersonic wind tunnels, the walls of the test section diverge at a slight angle with downstream distance. This is to allow for growth of the boundary layer and the effective change in area ratio with boundary layer displacement thickness. ${ }^{33}$ Thus, the change in pitot pressure with axial location will be a function of Mach number and Reynolds number, and so will not be the same for different operating conditions. A similar plot showing the change in Mach number with axial location is given in Fig. 23. With the exception of the lowest stagnation pressures, the Mach number gradient is generally less than 0.02 , or roughly $0.3 \%$, over the length of the test section.

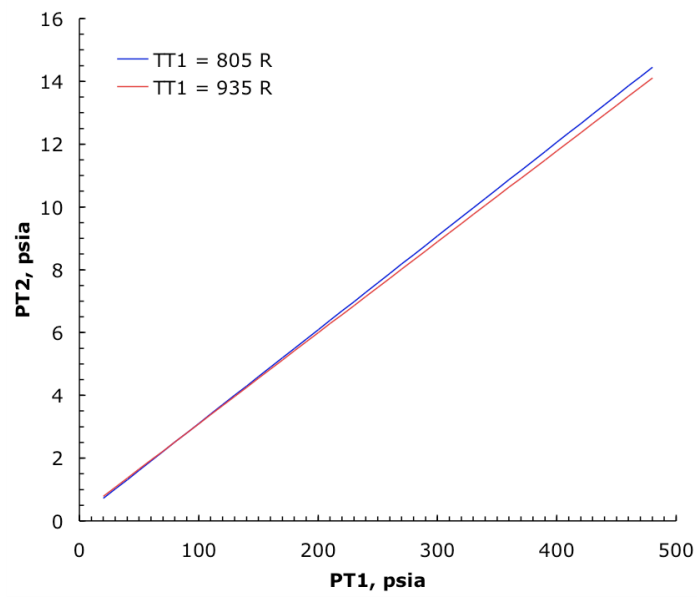

Figure 21. Effect of stagnation pressure and temperature on predicted pitot pressure.

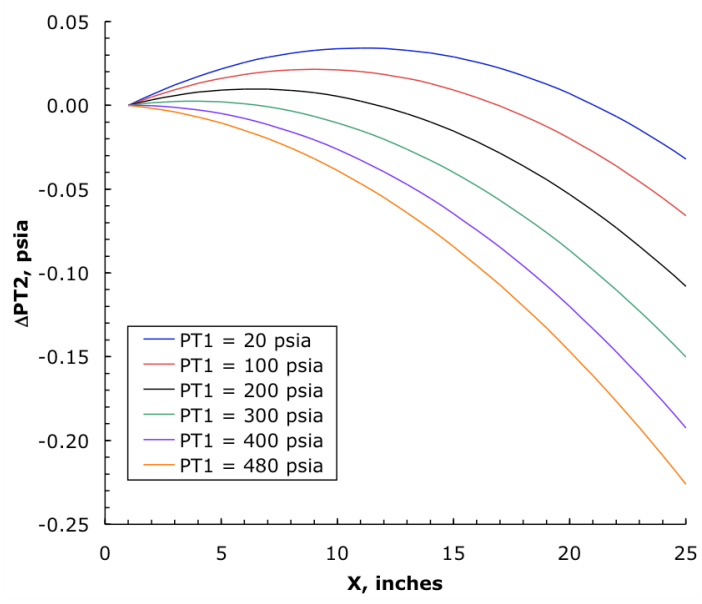

Figure 22. Variation in pitot pressure with axial location and stagnation pressure.

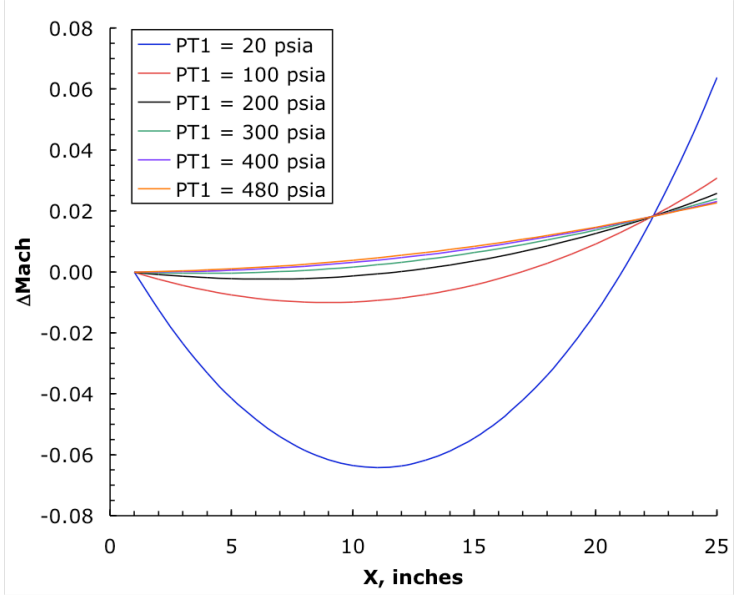

Figure 23. Variation in Mach number with axial location and stagnation pressure.

\section{F. Comparisons with Wall Pressure Measurements}

Wall pressures were measured at four different locations along the upper centerline of the test section, providing an independent set of measurements for comparisons of calculated Mach number. The recorded wall pressures, for both the design and confirmation points, at one station, $X=14.15$ inches, were used to compute Mach number from the ratio, $P_{w} / P_{T, l}$. These Mach numbers are compared in Fig. 24 to those derived from the calibration response 
model and the ratio, $P_{T, 2} / P_{T, 1}$, at the same pressure/temperature conditions and the same axial position. As seen in the graph, the Mach numbers derived from the two independent measurements are in very good agreement, both in the trends and magnitudes of the values. The outlier points seen in the wall-pressure data are likely due to interference from the pitot rake when at certain positions and orientations. The scatter seen in the regression model data is not due to "noise", but rather the varying stagnation temperatures among the points.

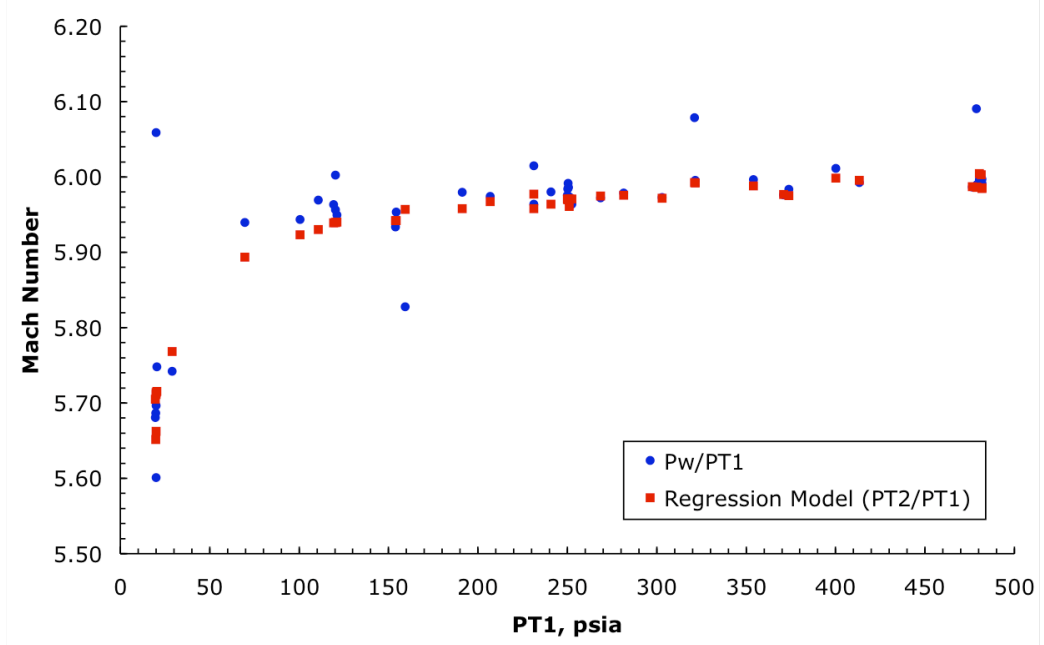

Figure 24. Comparison of Mach numbers computed from wall pressure ratio and regression model for $X=14.15$ inches.

\section{G. Comparisons with Previous Calibration Results}

An important aspect in reviewing results from a wind tunnel calibration, or any calibration for that matter, is determining whether some change has occurred in the behavior of the system since the last time it was checked. A significant change - one outside of the confidence intervals of the model - may indicate some problem that requires corrective action, such as a leak in the nozzle or degradation of the throat. In the present case, a direct comparison with previous results is somewhat limited by the prior confounding of the pressure and temperature effects as one was changed along with the other. Nonetheless, some comparison plots are presented to show the differences.

The change in $P_{T, 2}$ as a function of stagnation pressure is illustrated in Fig. 25. To remove any confounding temperature effect, the comparisons are made at the stagnation pressures and temperatures set for the previous calibration, but at the same test section axial location, $X=13$ inches. The agreement is generally good, although the difference (new minus old) in pitot pressure, plotted on the right side of the figure, reveals a shift in the slope of the response from the old calibration to the new. It is hard to say whether this shift represents a change in the facility or is simply a result of the way in which the two calibrations were performed. The 1995 calibration was run with factors changed one at a time while holding other factors constant. The sequential setting order of the independent variables would have allowed potential bias errors to creep into the results undetected. On the other hand, the use of randomization and blocking in the present calibration provided defense against such systematic variations. For the most part, the differences fall within the $95 \%$ confidence intervals for the 2005 calibration.

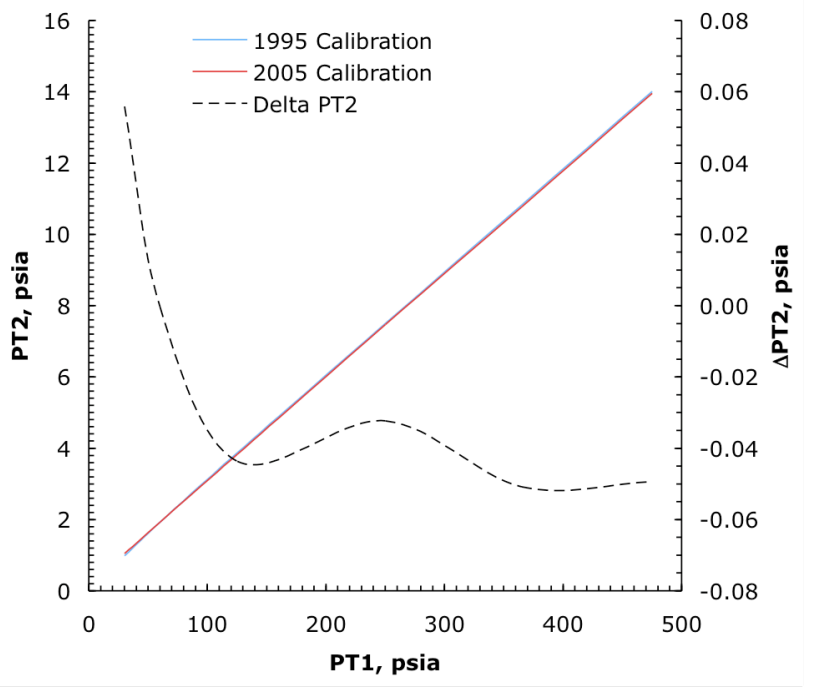

Figure 25. Comparison of pitot pressure trends from current and previous calibrations. 
The effects of test section axial location on the pitot pressure are shown in Fig. 26 at three different stagnation pressures for both the 1995 and 2005 calibrations. As before, the comparisons are made for identical stagnation temperature conditions. The change in $P_{T, 2}$ from its upstream limit value is plotted to allow the effect to be seen. The axial location is referenced to the 2005 calibration origin, and data are shown only for axial locations between 2.5 and 18.5 inches - the limits in the previous calibration. As seen in the figure, both calibrations show a quadratic response with axial location; however, the trends are somewhat different, particularly for the highest pressure. Again, these differences could be due to the manner in which the regression models were developed. With the prior calibration, the ratio, $P_{T, 2} / P_{T, 1}$, was fit as a quartic polynomial function of $P_{T, 1}$ for each separate axial location in the experiment. The coefficients of these quartic models were then fit as quadratic functions of the axial location. So the final calibration model was a quartic fit to $P_{T, I}$ with variable coefficients as functions of $X$. Such a model is not a regression fit to the data as a whole, but rather a set of individual, single-variable curve-fits to smaller subsets of the data, and therefore may not have fully characterized the changes in pitot pressure with axial location.

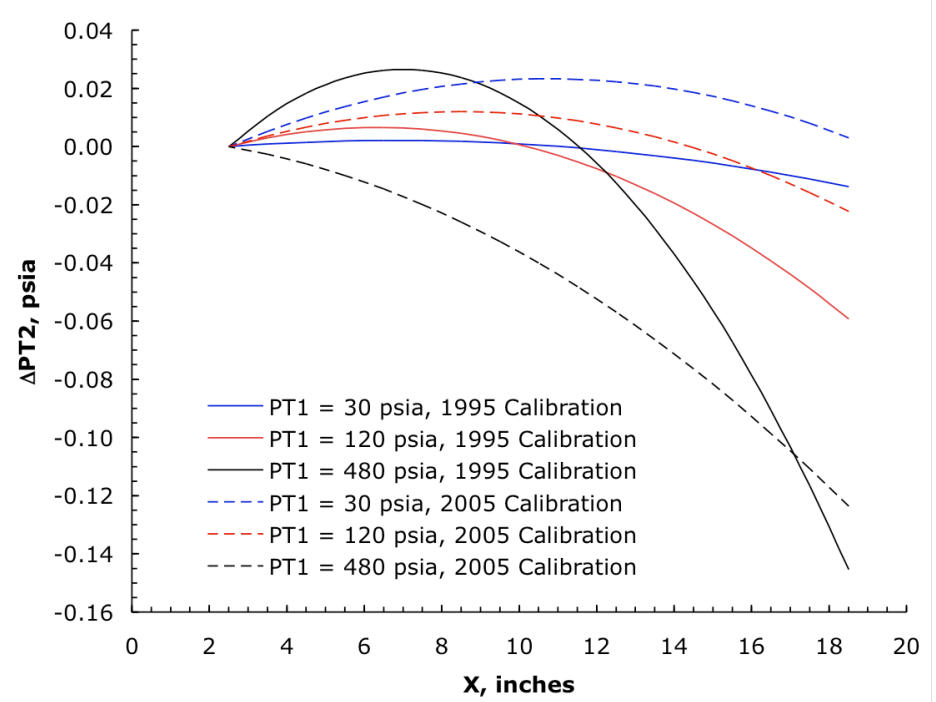

Figure 26. Comparison of axial location trends in pitot pressure from current and previous calibrations.

\section{Summary and Conclusions}

A calibration of the Langley 20-Inch Mach 6 Tunnel was performed to update the previous results and to expand the calibration over a wider range of the facility's operating envelope. Formal experiment design methods were used to develop a compact matrix of 34 runs leading to a quadratic response model of the pitot pressure as a function of the stagnation pressure and temperature, and the longitudinal location within the test section. Blocking, randomization, and replication were employed to defend against systematic errors and to reduce net random errors, while spatial randomization of the pitot rake roll orientation allowed broader sampling of the flowfield without increasing the data volume. Twelve separate, random independent variable combinations were run and confirmed the ability of the model to yield predictions to within the specified tolerance.

The structure of the experiment permitted the explicit capture of interactions between independent variables that were not expressed in prior calibration regression models. Comparisons to previous data were favorable, with the main differences seen in the effects of test section axial location. Present results show a strong axial Mach number gradient at the very lowest stagnation pressure, but for the majority of the stagnation pressure range, the Mach gradient is between 0.0009 and 0.0021 per inch. Mach numbers derived from pitot pressure and wall pressure measurements were in very good agreement, further confirming the accuracy of the model.

The pitot rake used in the experiment was existing equipment designed and built originally for use in a different facility. The design and construction of the rake made it difficult to physically move the rake, particularly the axial location in the test section. Given the randomization of the set point order, this inability to easily change the rake 
position slowed the test more than was expected. Future calibration experiments will utilize hardware designed with randomization in mind so that the resource savings provided by formally designed experiments can be fully realized.

The experiment resulted in a regression model that describes the functional relationship between the pitot pressure and the independent variables. The coefficients of this model might serve as suitable measurands for statistical process control (SPC). In a SPC framework, each coefficient in the response model would be tracked on a separate process control chart; with control limits established after an appropriate amount of data had been acquired. ${ }^{34}$ Future calibrations would then be judged on whether or not the model coefficients fell within their respective limits. Failure to meet the tolerance levels would indicate a possible change in the performance of the facility or a lack of longterm stability in the measurement system. In the situation where the coefficients do remain within the limits over time, the time-averaged mean would represent a truer value of the coefficient, with the control limits providing better estimates of the confidence intervals for each coefficient.

The present calibration experiment was performed in a total of 46 runs, not counting those necessary to "shake down" the experiment. This total is far less than is usually encountered in a facility calibration. The success of the experiment in producing a usable and accurate response model demonstrates that good calibrations need not be so resource intensive. The use of MDOE has the potential to facilitate more frequent and more accurate wind tunnel calibrations, improving the precision of the known flow conditions.

\section{Acknowledgments}

The authors would like to thank Richard Wheless of NCI Information Systems for his help in preparing illustrations for this manuscript. Also acknowledged for their efforts are the engineers and technicians of the 20-Inch Mach 6 Tunnel: Teck-Seng Kwa, Grace Gleason, Buddy Stotler, and Roland Hatten. Dr. Ramadas Prabhu is recognized for his timely computations of the flow around pitot tubes. Finally, a special thanks goes to Anthony Robbins for his inestimable help in setting up the hardware and instrumentation for this experiment.

\section{References}

${ }^{1}$ Guide to the Expression of Uncertainty in Measurement, International Organization for Standardization, 1995.

${ }^{2}$ Assessment of Experimental Uncertainty with Application to Wind Tunnel Testing, AIAA Standard S-071A-1999, 1999.

${ }^{3}$ Cahill, D.M., "Development of an Uncertainty Methodology for Multiple-Channel Instrumentation Systems," AIAA Paper 923953, July 1992.

${ }^{4}$ Cahill, D.M., "Application of Uncertainty Methodology for Wind Tunnel Facilities at AEDC," AIAA Paper 96-2216, June 1996.

${ }^{5}$ Meyn, L.A., "Software Tools for Measurement Uncertainty Analysis,” AIAA Paper 98-2713, June 1998.

${ }^{6}$ Coleman, H.W., and Steele, W.G., Experimentation and Uncertainty Analysis for Engineers, John Wiley \& Sons, New York, 1989, Chapters 3, 4.

${ }^{7}$ Kammeyer, M.E., "Wind Tunnel Facility Calibrations and Experimental Uncertainty,” AIAA Paper 98-2715, June 1998.

${ }^{8}$ Hemsch, M.J., "Development and Status of Data Quality Assurance Program at NASA Langley Research Center - Towards National Standards,” AIAA Paper 96-2214, June 1996.

${ }^{9}$ Hemsch, M.J., "Langley Wind Tunnel Data Quality Assurance - Check Standard Results,” AIAA Paper 2000-2201, June 2000.

${ }^{10}$ DeLoach, R., "Applications of Modern Experiment Design to Wind Tunnel Testing at NASA Langley Research Center," AIAA Paper 98-0713, January 1998.

${ }^{11}$ DeLoach, R., "Improved Quality in Aerospace Testing Through the Modern Design of Experiments," AIAA Paper 2000-0825, January 2000.

${ }^{12}$ Keyes, J.W., "Force Testing Manual for the Langley 20-Inch Mach 6 Tunnel,” NASA TM 74026, July 1977.

${ }^{13}$ Micol, J.R., "Langley Aerothermodynamic Facilities Complex: Enhancements and Testing Capabilities," AIAA Paper 98-0147, January 1998.

${ }^{14}$ Strike, W.T., Coulter, S.M., and Mills, M.L., "Calibrations of the AEDC-VKF Hypersonic Wind Tunnels at Nozzle Mach Numbers 6, 8, and 10,” AEDC-TR-92-14, January 1993.

${ }^{15}$ Pope, A., and Goin, K.L., High-Speed Wind Tunnel Testing, John Wiley \& Sons, New York, 1965, Chapters 10, 11.

${ }^{16}$ Ames Research Staff, "Equations, Tables, and Charts for Compressible Flow," NACA TR-1135, 1953.

${ }^{17}$ Montgomery, D.C., Design and Analysis of Experiments, $5^{\text {th }}$ ed., John Wiley \& Sons, New York, 2001, Chapters 3, 11, 13.

${ }^{18}$ DeLoach, R., "Tailoring Wind Tunnel Data Volume Requirements Through the Formal Design of Experiments," AIAA Paper 98-2884, June 1998.

${ }^{19}$ Boudreau, A.H., "Performance and Operational Characteristics of AEDC/VKF Tunnels A, B, and C," AEDC-TR-80-48, July 1981.

${ }^{20}$ Montgomery, D.C, and Runger, G.C., Applied Statistics and Probability for Engineers, $2^{\text {nd }}$ ed, John Wiley \& Sons, New York, 1999, Chapter 8.

${ }^{21}$ Box, G.E., and Draper, N.R., Empirical Model-Building and Response Surfaces, John Wiley \& Sons, New York, 1987. 
${ }^{22}$ Myers, R.H., and Montgomery, D.C., Response Surface Methodology: Process and Product Optimization Using Designed Experiments, $2^{\text {nd }}$ ed., John Wiley \& Sons, 2002.

${ }^{23}$ DeLoach, R., and Erickson, G.E., "Low-Order Response Surface Modeling of Wind Tunnel Data Over Truncated Inference Subspaces," AIAA Paper 2003-0456, January 2003.

${ }^{24}$ DeLoach, R., "Tactical Defenses Against Systematic Variation in Wind Tunnel Testing," AIAA Paper 2002-0885, January 2002.

${ }^{25}$ DeLoach, R., "Blocking: A Defense Against Long-Period Unexplained Variance in Aerospace Ground Testing," AIAA Paper 2003-0650, January 2003.

${ }^{26}$ Micol, J.R., Midden, R.E., and Miller, C.G., "Langley 20-Inch Hypersonic CF $_{4}$ Tunnel: A Facility for Simulating Real-Gas Flows," AIAA Paper 92-3939, July 1992.

${ }^{27}$ Bibb, K.L, Peraire, J., and Riley, C.J., "Hypersonic Flow Computation on Unstructured Meshes," AIAA Paper 97-0625, January 1997.

${ }^{28}$ Potter, J.L, and Bailey, A.B., "Pressures in the Stagnation Regions of Blunt Bodies in the Viscous-Layer to Merged-Layer Regimes of Rarified Flow," AEDC-TDR-63-168, September 1963.

${ }^{29}$ Peterson, C.W., and George, O.L., "Wind Tunnel Pressure Probes: New Calibrations for New Geometries and Flow Environments," AIAA Paper 74-635, July 1974.

${ }^{30}$ Hollis, B.R., "Real-Gas Flow Properties for NASA Langley Research Center Aerothermodynamic Facilities Complex Wind Tunnels," NASA CR 4755, September 1996.

${ }^{31}$ Draper, N.R., and Smith, H., Applied Regression Analysis, $3^{\text {rd }}$ ed., John Wiley \& Sons, New York, 1998, Chapter 23.

${ }^{32}$ Anderson, J.D., Jr., Hypersonic and High-Temperature Gas Dynamics, McGraw-Hill, New York, 1989, Chapter 6.

${ }^{33}$ Lukasiewicz, J., Experimental Methods of Hypersonics, Marcel Dekker, New York, 1973, Chapter 8.

${ }^{34}$ Wheeler, D.J., and Chambers, D.S., Understanding Statistical Process Control, $2^{\text {nd }}$ ed., SPC Press, Knoxville, TN., 1992. 